\title{
REVIEW
}

\section{Nanowire-supported plasmonic waveguide for remote excitation of surface-enhanced Raman scattering}

\author{
Yingzhou Huang ${ }^{1,2, *}$, Yurui Fang ${ }^{3, *}$, Zhenglong Zhang ${ }^{4,5, *}$, Ling Zhu ${ }^{6}$ and Mengtao Sun ${ }^{2,1}$
}

Due to its amazing ability to manipulate light at the nanoscale, plasmonics has become one of the most interesting topics in the field of light-matter interaction. As a promising application of plasmonics, surface-enhanced Raman scattering (SERS) has been widely used in scientific investigations and material analysis. The large enhanced Raman signals are mainly caused by the extremely enhanced electromagnetic field that results from localized surface plasmon polaritons. Recently, a novel SERS technology called remote SERS has been reported, combining both localized surface plasmon polaritons and propagating surface plasmon polaritons (PSPPs, or called plasmonic waveguide), which may be found in prominent applications in special circumstances compared to traditional local SERS. In this article, we review the mechanism of remote SERS and its development since it was first reported in 2009. Various remote metal systems based on plasmonic waveguides, such as nanoparticle-nanowire systems, single nanowire systems, crossed nanowire systems and nanowire dimer systems, are introduced, and recent novel applications, such as sensors, plasmon-driven surface-catalyzed reactions and Raman optical activity, are also presented. Furthermore, studies of remote SERS in dielectric and organic systems based on dielectric waveguides remind us that this useful technology has additional, tremendous application prospects that have not been realized in metal systems.

Light: Science \& Applications (2014) 3, e199; doi:10.1038/lsa.2014.80; published online 15 August 2014

Keywords: nanowire; plasmonic waveguide; remote-excitation; surface-enhanced Raman scattering

\section{INTRODUCTION}

As a branch of nano-optics, plasmonics has attracted the increasing interest of researchers worldwide with the rapid development of nanofabrication in recent decades. ${ }^{1-3}$ The most amazing property of plasmonics is that it can manipulate light at subdiffraction wavelengths and hence opens interesting avenues to study light-matter interactions at the nanoscale. ${ }^{4}$ The collective oscillation of free electrons at a metal surface is called surface plasmons. When excited by light, the hybridized excitations of surface plasmons and coupled photons are called surface plasmon polaritons (SPPs). Because SPPs can confine the energy of an electromagnetic (EM) field near the metal surface and decay exponentially with vertical distance, two fundamental excitations of SPPs, propagating SPPs (PSPPs, plasmonic waveguide) and localized SPPs, have tremendous application prospects. The former could overcome the traditional diffraction limit in dielectric optics and be the key approach to overcoming the bottleneck of the miniaturization of nanophotonic devices and large-scale on-chip integrated circuits for next-generation information technology. ${ }^{5-11}$ The extremely enhanced EM field caused by the latter has great application values in various fields, such as surface-enhanced spectrum, ${ }^{12-15}$ surface plasmon resonance sensors, ${ }^{16-19}$ ultra transmission, ${ }^{20,21}$ plasmonic trapping, ${ }^{22,23}$ plasmonic-enhanced emission, ${ }^{24,25}$ quantum communication, ${ }^{26,27}$ super-resolution microscopy, ${ }^{28}$ cloaking, ${ }^{29}$ photothermal cancer therapy, ${ }^{30,31}$ steam generation, ${ }^{30,32,33}$ holography, ${ }^{34}$ photovoltaics $^{35-37}$ and water splitting. ${ }^{38-40}$

One of the most promising applications of SPPs, especially localized SPPs, is surface-enhanced Raman scattering (SERS), which has been studied both theoretically and experimentally for many decades. ${ }^{12-14,41-57}$ As an optical fingerprint, Raman spectra, which could be used to analyze chemical structures, are widely used in scientific investigations and material analysis. However, as a weak process, the small Raman scattering cross-section makes the detection of a small number of molecules a problem even after the laser power is increased. An up to $10^{10}-10^{11}$ enhancement over the typical Raman scattering crosssection in SERS solves this problem and enables the detection of Raman spectra at the single molecule level, which is commonly performed at noble metal surfaces such as rough surfaces or nanoparticle aggregates. ${ }^{13,14}$ The dominate contribution to this enhancement is generally thought to be the EM fields greatly enhanced by localized SPPs. ${ }^{12,52,58}$ In addition, the charge transfer processes, which are the

${ }^{1}$ Soft Matter and Interdisciplinary Research Center, College of Physics, Chongqing University, Chongqing 400044, China; ${ }^{2}$ Beijing National Laboratory for Condensed Matter Physics, Institute of Physics, Chinese Academy of Sciences, Beijing 100190, China; ${ }^{3}$ Division of Bionanophotonics, Department of Applied Physics, Chalmers University of Technology, Göteborg 41296, Sweden; ${ }^{4}$ Leibniz Institute of Photonic Technology, 07745 Jena, Germany; ${ }^{5}$ Physical Chemistry, Friedrich-Schiller University Jena, 07743 Jena, Germany and ${ }^{6}$ National Center for Nanoscience and Technology, Beijing 100190, China

*These authors contributed equally to this work

Correspondence: Professor MT Sun, Beijing National Laboratory for Condensed Matter Physics, Institute of Physics, Chinese Academy of Sciences, Beijing 100190, China

E-mail: mtsun@iphy.ac.cn

Or Professor YZ Huang, Soft Matter and Interdisciplinary Research Center, College of Physics, Chongqing University, Chongqing 400044, China

E-mail: yzhuang@cqu.edu.cn

Received 20 March 2014; revised 14 May 2014; accepted 14 May 2014 
electronic structure variation of molecules adsorbed on metal surfaces, also play an important role in the SERS effect. ${ }^{45-48,54}$ The area where the highest EM field enhancement is generated in a nanostructure is called a 'hot spot', such as the nanogap between the nanoparticle dimer and the gaps between nanoparticles and wires. The Raman signals in most SERS experiments are obtained at the tiny hot spots. ${ }^{12}$

In this article, we review the mechanism and development of remote SERS since it was first reported in 2009, ${ }^{59,60}$ and discuss several advantages compared to traditional SERS that may find prominent applications in special circumstances. In a traditional SERS, the incident light is directly focused on the detecting target, which is called 'direct mode' or local SERS. In contrast, the 'remote SERS' refers to the illumination spot of incident light that is far away from the target, and excited by the energy guided by the PSPPs in a 'remote mode'. The energy transfer between the illumination spot and the hotspot is mainly supported by the plasmonic waveguide (PSPPs) in various remote systems, as illustrated in Figure 1. PSPPs are the propagation of charge-density waves at the metal/dielectric interface, which emit photons into the free space at defects such as nanoparticle-wire gaps and the terminal of the wire, while some of the energy is lost as ohmic damping in the metal. PSPPs could of course excite the hot spot remotely. ${ }^{5}$ The remotely excited hot spot is of nanoscale dimensions; it avoids background noise due to a large excitation area created by the incident illumination spot, isolates the heat from the focused incident light area to the target illumination in the subdiffraction wavelength area and, naturally, reduces the possibility of damage to the sample caused by the high laser intensity. The remote SERS provides a novel way to perform sensing measurements based on surface-enhanced optical signals and may have applications in systems where traditional SERS is unsuitable, such as the Raman detection of biomolecules in vivo in cells.

Because of their good capability as plasmonic waveguides, chemically synthesized metal nanowires with micrometer-scale lengths and nanoscale diameters play a primary role in most previous reports of remote SERS. In this review, we first illustrate the synthesis and fabrication of metallic nanowires and the corresponding physical mechanism of the plasmonic waveguide. Second, various remote metal systems, such as a nanoparticle-nanowire system, ${ }^{60}$ single nanowire system, ${ }^{61}$ crossed nanowires system, ${ }^{62}$ nanowire dimer system ${ }^{63}$ and

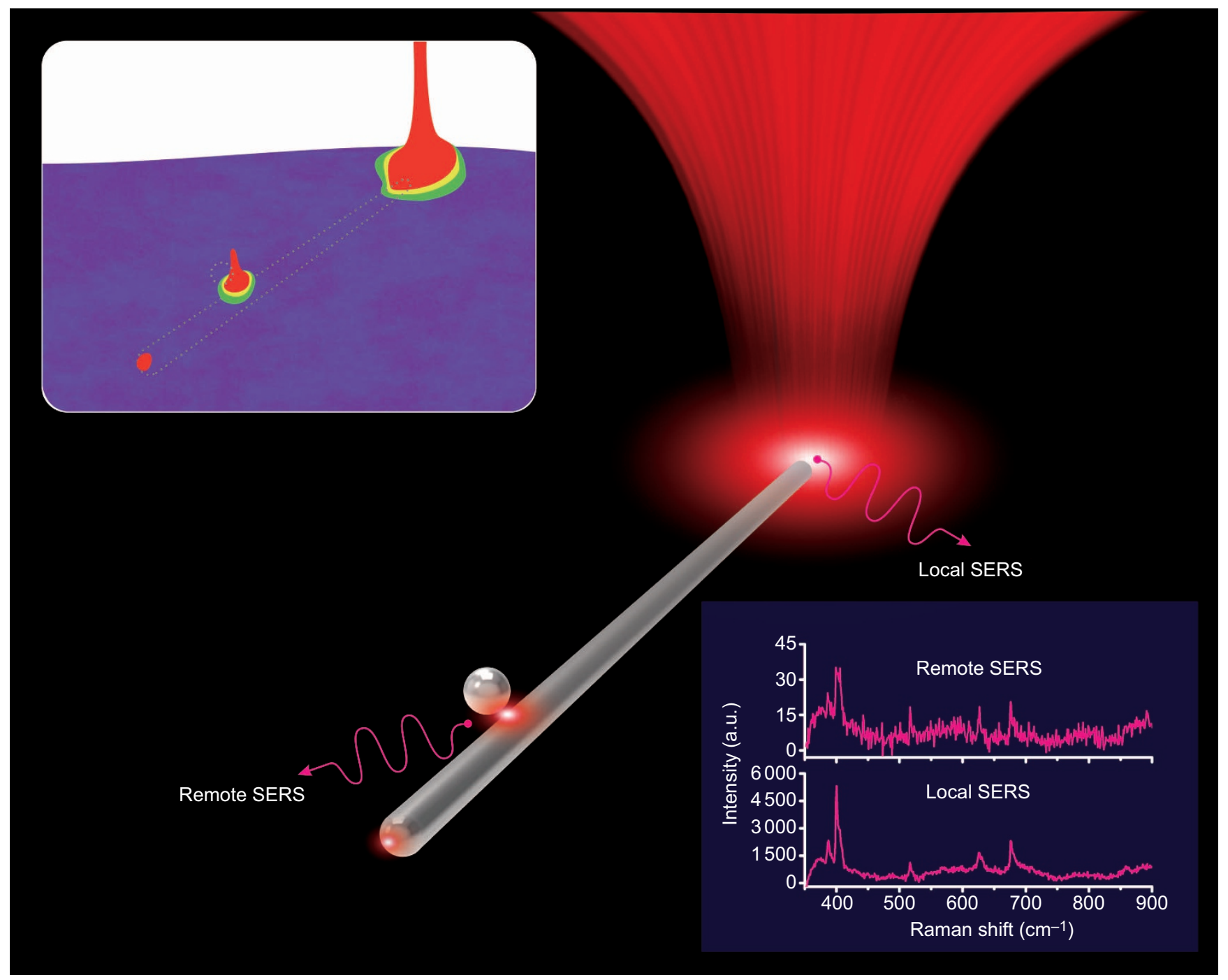

Figure 1 A sketch of the local SERS and the remote SERS in an Ag nanoparticle-nanowire system (Raman molecule adsorbed on the Ag nanowire). The Raman signals obtained at the illuminating terminal are local SERS, while the signals collected at the junction whose energy come from the propagating SPPS are remote SERS. The insets are the three-dimensional distribution of the Raman intensity and spectra. SERS, surface-enhanced Raman scattering; SPP, surface plasmon polariton. 
nanowire bundle system, ${ }^{64}$ as well as a remote semiconductor system $^{49,65}$ and a remote organic system, ${ }^{66}$ are presented. Third, fields of applications of remote SERS, such as sensitive sensors for in vivo cells, ${ }^{59,60,67}$ plasmon-driven surface-catalyzed reactions ${ }^{62,68,69}$ and Raman optical activity, ${ }^{70}$ are introduced. Finally, we summarize this article and give a tentative outlook.

\section{MECHANISM OF A PLASMONIC WAVEGUIDE ON METAL NANOWIRES}

To efficiently couple light into and out of PSPPs by compensating for the momentum difference between light and the PSPPs, various metal nanostructures have been investigated in previous reports, ${ }^{5}$ such as nanogrooves, ${ }^{71}$ nanoparticle chains ${ }^{72}$ and nanowires. ${ }^{73,74}$ Among them, the chemically synthesized noble metal nanowires with subdiffraction-limited diameters may be a preferable structure because of their smooth crystalline surface, which is unachievable by other physical nanofabrication methods, such as electron beam lithography and extreme ultraviolet lithography. On such wires, the SPPs could propagate over tens of microns with relative low energy loss in the visible spectrum and even longer in the infrared. ${ }^{5,10,75-79}$ Therefore, most of the remote systems in remote SERS experiments adopt gold or silver nanowire systems, as in the previous report.

\section{Synthesis and fabrication of metal nanowires}

Because of the smooth surface on the atomic scale and good crystal form, chemically synthesized single crystal noble nanowires exhibit improved capability as plasmonic waveguides compared to polycrystalline nanowires fabricated by lithography. ${ }^{74}$ Among noble metal nanowires, the Ag nanowire synthesized by the polyol method ${ }^{80}$ played a key role in plasmonic waveguides due to its small dissipative loss. In this method, silver nitrate was reduced into a nanowire by heated ethylene glycol and poly (vinyl pyrrolidone), which were both acting as reducing and capping agents. The length of the produced Ag nanowire was several to hundreds of micrometers, and the diameter was tens to hundreds of nanometers, which could be controlled by the reaction condition. ${ }^{80}$ Considering the low coupling efficiency of very thin nanowires $(<50 \mathrm{~nm})$ and the propagating length, the $\mathrm{Ag}$ nanowires with diameters of 100-250 $\mathrm{nm}$ and lengths of 10-20 $\mu \mathrm{m}$ played key roles in the studies of plasmonic waveguides. In addition to chemical synthesis, nanowires fabricated by electron beam lithography showed the capability for use as plasmonic waveguides, with a nearly $10-\mu \mathrm{m}$ propagation distance in an Ag-branched nanowire under 780-nm laser excitation. ${ }^{81}$ Despite the lesser capability of these plasmonic waveguides compared to those produced by chemical synthesis, nanowires fabricated by electron beam lithography have much more extensive application prospects due to their ability of precise modulation.

\section{Mechanism of a plasmonic waveguide}

PSPPs on metal nanowires could be easily excited by illumination at one terminal with an evanescent field produced by total internal reflection or a simply focused laser beam. ${ }^{5,73,74}$ The momentum mismatch requires the conversion between photon and SPP and only occurs at a position of symmetry breaking, such as terminals or defects. Therefore, the light emits at the distal terminal once the ohmic-damped PSPPs arrive. This plasmonic waveguide on metal nanowires could be understood by the Fabry-Pérot resonances because the PSPPs could also be reflected back at the terminal and interfere with the incoming PSPPs to form standing waves. ${ }^{75,82}$ The emission intensity at the distal terminal is dominated by both incident light and nanostructures, which affect the coupling efficiency and ohmic damping. The distribution of the near field on the nanowire and the periodic fluctuation of intensity exhibited in the emission spectrum in Figure 2 showed the wavelength dependence of the incident light, which confirmed the Fabry-Pérot resonance behavior. ${ }^{74,75}$ The polarization dependence of incident light in a plasmonic waveguide has also been studied for both $\mathrm{Ag}$ and Au nanowires. ${ }^{61,76}$

All of the results showed that the emission was greatly affected by the incident polarization and that a significantly larger propagation distance was achieved by illuminating light with the polarization parallel to the nanowire (Figure 3). This result is because multiple PSPP modes with different propagating efficiency were produced in the plasmonic waveguide in a thick nanowire. Different SPP modes and their excitation conditions are indicated in Figure 4. The incident light, whose polarization is represented by the angle $\theta$, is a paraxial Gaussian beam with an instantaneous electric field of the form $E_{\text {inc }}=E_{0} e^{-i \phi}$, where $E_{0}$ and $\phi$ are the mode profile and phase of the incident light, respectively. For the polarization of incident light parallel to the nanowire $\left(\theta=0^{\circ}\right)$, the fundamental $m=0$ SPP mode (charge oscillations along the wire
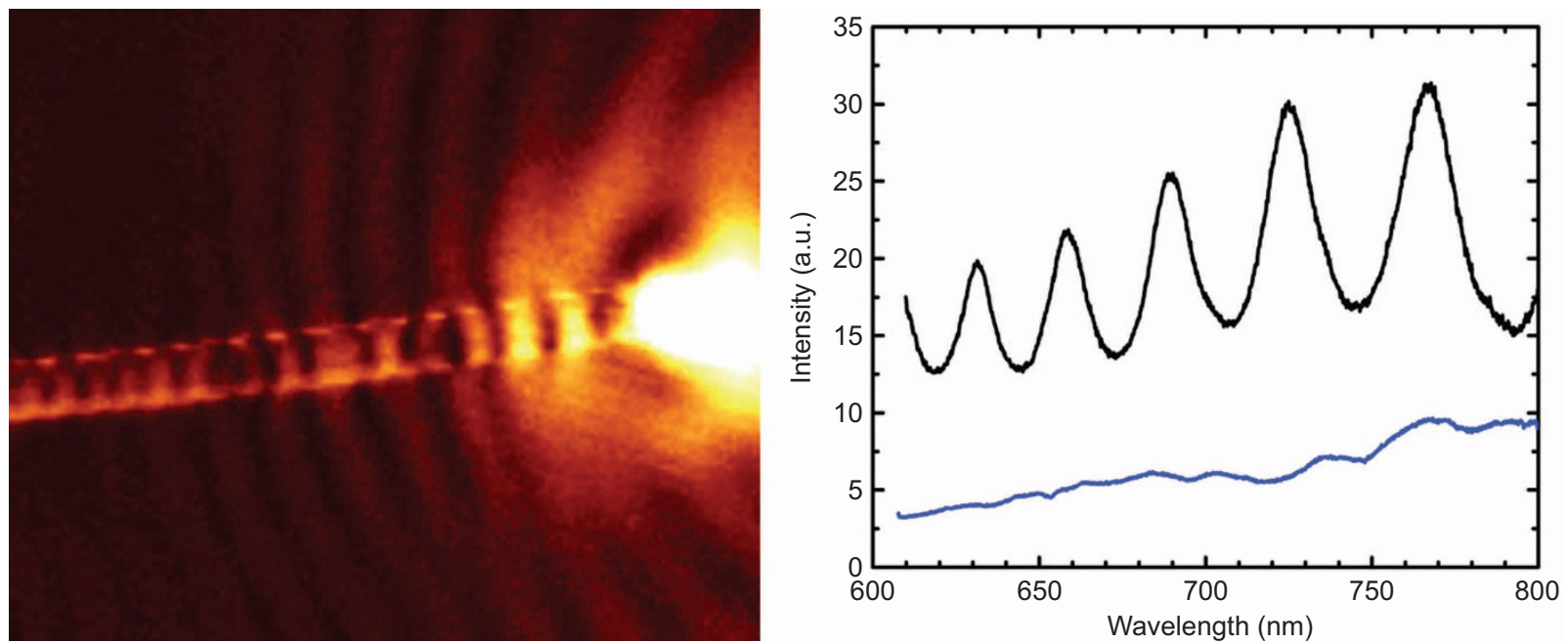

Figure 2 The near field distribution of the plasmonic waveguide in the Ag nanowire and the corresponding emission spectrum. Figure reproduced with permission: Ref. 74, ( ) 2005, APS. 

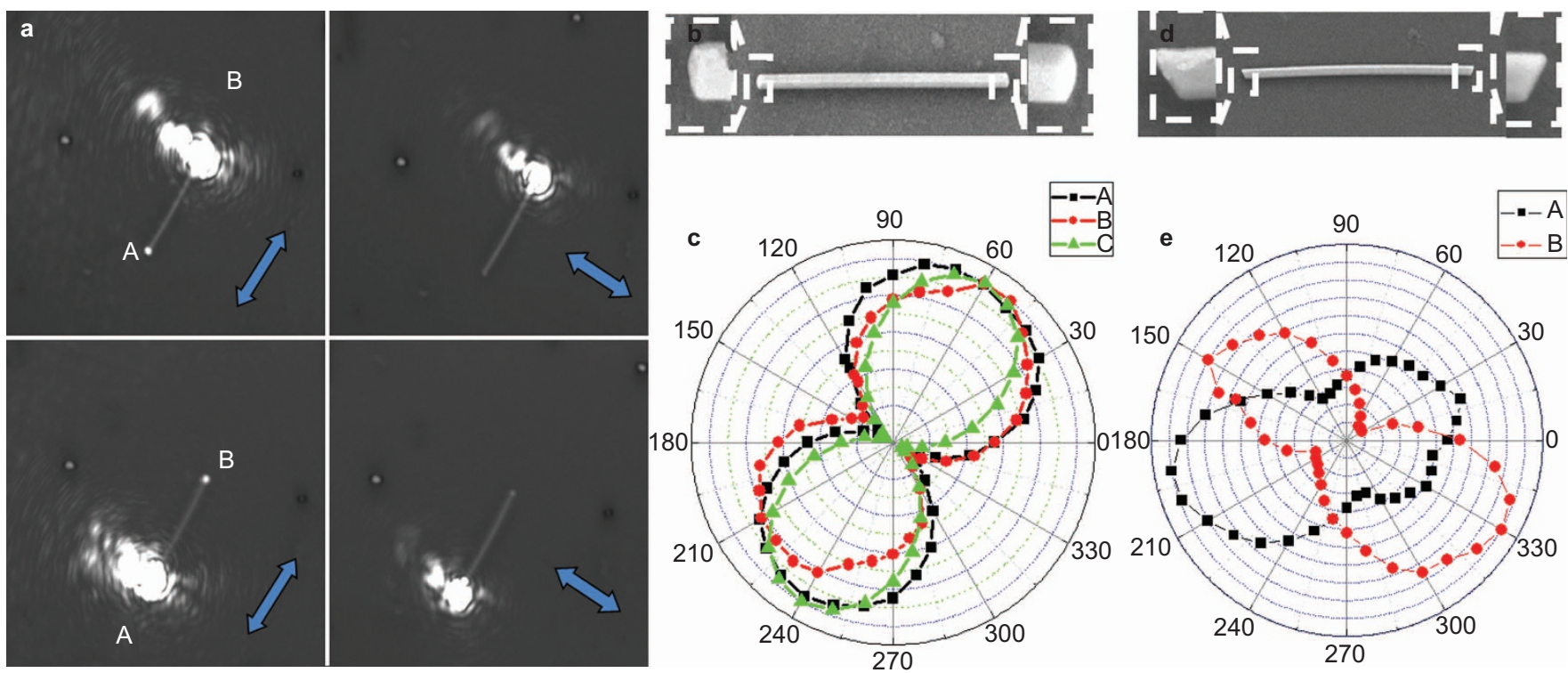

Figure 3 (a) An optical image of the plasmonic waveguide in a single Au nanowire. (b, c) An SEM image of a Au nanowire and the corresponding emission intensity with various incident polarizations. (d, e) An SEM image of another Au nanowire with a different geometric shape of the terminal and the corresponding emission intensity with various incident polarizations (blue arrow in a). Figure reproduced with permission: Ref. 61, (c) 2011, ACS. SEM, scanning electron microscopy.

axis) or $m=-1$ SPP mode (charge oscillations in the vertical plane) are selectively excited by aligning the input phase to $\phi=0$ or $\phi=\pi / 2$. For the polarization of incident light perpendicular to the nanowire $\left(\theta=90^{\circ}\right)$, the $m=1$ (charge oscillations in the horizontal plane) and $m=2$ SPP mode are generated for $\phi=0$ and $\phi=\pi / 2$, respectively. ${ }^{9,83}$

The structures of the nanowire also play important roles in the plasmonic waveguide, such as the diameter and the geometrical shape of the terminal. The shape of the illuminated terminal or the emission terminal has a significant effect on the in-coupling and out-coupling efficiency and results in a spatial distribution and polarization vari-

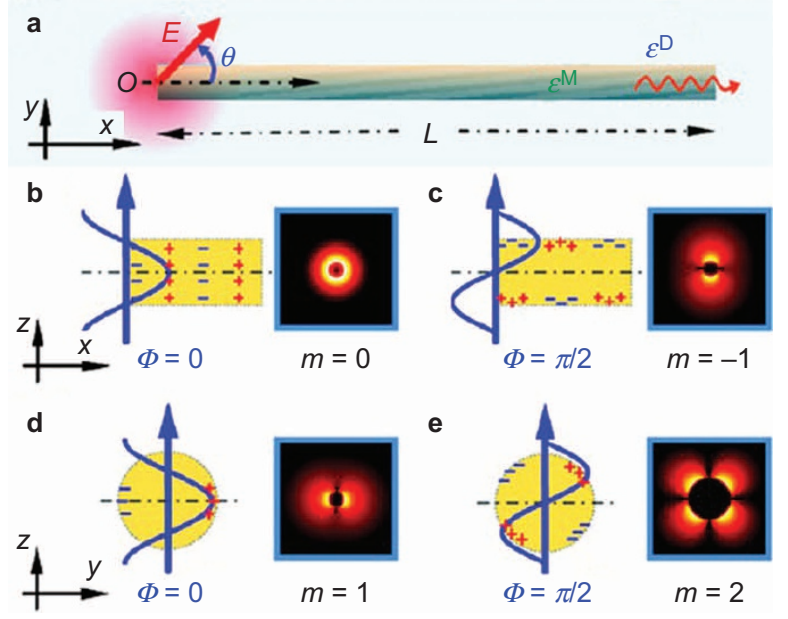

Figure 4 (a) The excitation of various SPP modes in a metal nanowire, where $\theta$ is the angle between the incident polarization and nanowire axis. (b) Parallel excitation ( $\theta=0^{\circ}$ ) at incident phases $\phi=0$ for the $m=0$ SPP mode. (c) Parallel excitation $\left(\theta=0^{\circ}\right)$ at incident phases $\phi=\pi / 2$ for the $m=-1$ SPP mode. (d) Perpendicular excitation $\left(\theta=90^{\circ}\right)$ at incident phases $\phi=0$ for the $m=1$ SPP mode. (e) Perpendicular excitation $\left(\theta=90^{\circ}\right)$ at incident phases $\phi=\pi / 2$ for the $m=2$ SPP mode. The inset is the $E$-field profile of each SPP mode. Figure reproduced with permission: Ref. 9, (c) 2011, APS. SPP, surface plasmon polariton. ation in the emission. ${ }^{61,76}$ The diameter of the nanowire has a direct relation to the excited multiple SPP modes in that a thicker nanowire could support additional high-order SPP modes. ${ }^{9}$ Interestingly, for the nanowires that could only support the three lowest SPP modes $(|m| \leq 1)$, as shown in Figure 5, the plasmonic waveguide would exhibit a helix behavior. ${ }^{6,9}$ Due to a constant phase delay of $\pi / 2$ between the $m=1$ and $m=-1$ SPP modes, a circularly polarized prop-
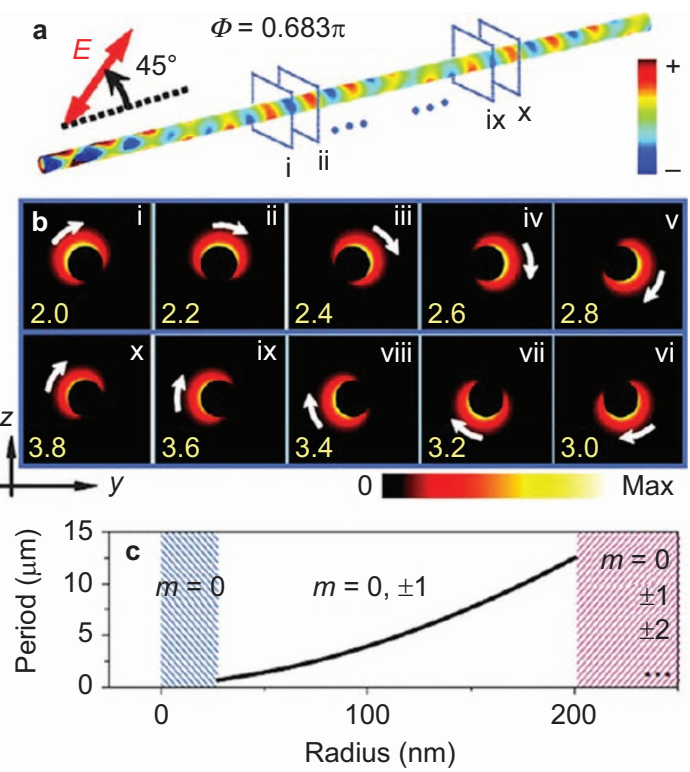

Figure 5 Helix plasmonic waveguide in the Ag nanowire. (a) Distribution of the surface charge density. (b) Variation of the time-averaged power flow in the vertical plane along the nanowire axis. (c) The excitation of the SPP modes and the period of the SPP helix with various nanowire radii. There is no helix in the single SPP mode regime (blue area) and multi-SPP mode regime (magenta area). Figure reproduced with permission: Ref. 9, ( 2011 , APS. SPP, surface plasmon polariton. 
agating SPP could be generated by the coherent interference when the two SPP modes have equal amplitude. This circularly propagating SPP is stretched by the simultaneously excited $m=0$ SPP mode into a helical SPP wave at the nanowire surface.

The time-averaged power flow and surface charge distribution in Figure 5 illustrate the energy of the electromagnetic field near the nanowire at the metal/dielectric interface in the plasmonic waveguide. A larger helical period is generated on a thicker nanowire because the propagation constant of the $m= \pm 1$ SPP mode increases and the $m=0$ SPP mode decreases, ${ }^{83}$ resulting in the formation of beating period variations (Figure $5 \mathrm{c}$ ). For very thin nanowires (diameter $D<50 \mathrm{~nm}$ ), there is only the $m=0$ SPP mode, and the helical behavior disappears. For very thick nanowires $(D>400 \mathrm{~nm})$, complicated field distributions are generated for the excitation of higher order SPP modes $(m \geqslant 2)$, which countermand the helix plasmonic waveguide. The large tenability of the plasmonic waveguide in the metal nanowire is also caused by other factors, such as the spacer layer between the nanowire and the substrate, surrounding dielectrics and the bending angle of the nanowire. In the plasmonic waveguide, a coupling between the PSPPs and the photonic modes of the substrate occurs and is dominated by the thickness of the spacer. The relation could be illustrated by the following formula: ${ }^{82}$

$$
I_{e}=I_{0} C(d) e^{-L / \gamma(D, d)}
$$

where $I_{0}$ is the intensity of the incident light, $d$ is the thickness of the spacer layer between the substrate and the nanowire, $C(d)$ is the coupling coefficient of the light to the SPPs, $L$ is the length of the nanowire, $D$ is the diameter of the nanowire and $r(D, d)$ is the $1 / e$ PSPP damping length. The variation in the coupling efficiency is due to the interference between the incident light and the light reflected by the substrate. Both the formula and the experimental results in Figure 6 indicate that the average emission intensity varies with the spacer thickness, even for nanowires of the same length. Similar to the metal nanoparticle and the metal surface, the properties of the SPP in a plasmonic waveguide on a metal nanowire are largely modulated by the surrounding dielectrics. The beat period of the near-field distribution at the nanowire surface, which was generated by the coherent interference of the two lowest SPP modes, was found to increase by $90 \mathrm{~nm}$ per nanometer of $\mathrm{Al}_{2} \mathrm{O}_{3}$ coating or by $16 \mu \mathrm{m}$ per refractive index unit variation in the surrounding dielectric medium (Figure 6). ${ }^{8}$ When the wire is not straight, there will be an energy attenuation purely due to bending, which follows an exponential function of the bending radius. If it is simply assumed that the intensity of the emission at the bend terminal decays exponentially with the curved length of the nanowire, but with a different attenuation coefficient related to the bending radius, the pure bending loss could be mathematically calculated (Figure 7). ${ }^{84}$ Even including the loss, both straight and bending wire can support PSPPs and transfer energy to remote locations.

\section{REMOTE SYSTEMS}

\section{Nanoparticle-nanowire metal system}

The localized SPPs and PSPPs on noble metal nanoparticle-nanowire systems have been widely studied in the past. ${ }^{58,85}$ The nanogap between a nanoparticle and a nanowire could be the hotspot of SERS for a sensitive sensor. In addition, a disruption point in this system, such as a sharp corner or the terminals in the nanowire, or a nanoparticle-adjoined nanowire could be the position for the efficient coupling of light into and out of PSPPs. ${ }^{5,86}$ Therefore, the Ag nanoparticle-nanowire system was adopted in the first report of remote SERS in $2009 .{ }^{59,60}$ In the report by Fang and co-workers, ${ }^{60}$ malachite green isothiocyanate (MGITC) was used as a Raman molecule at the single-molecule level in the Ag nanoparticle-nanowire junction that
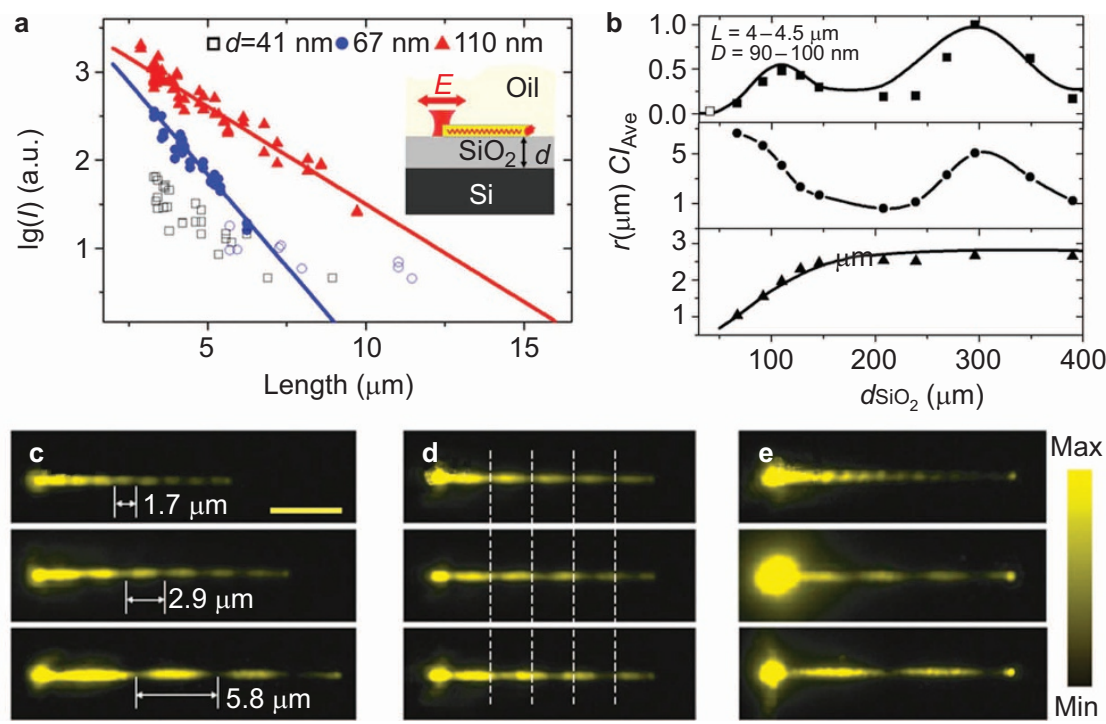

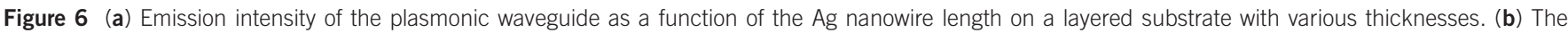

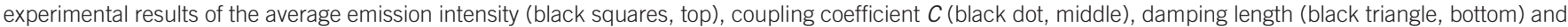

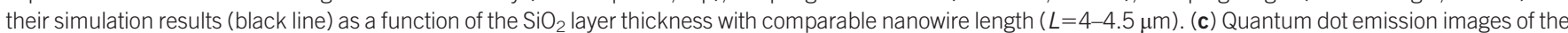

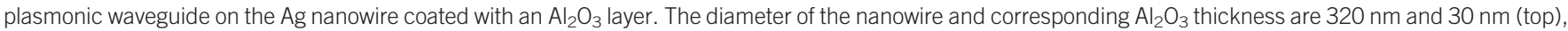

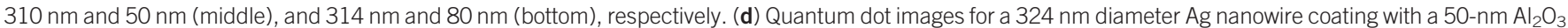

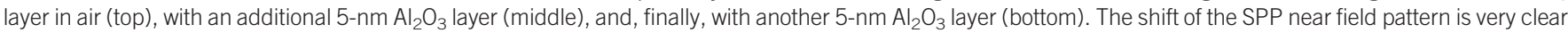

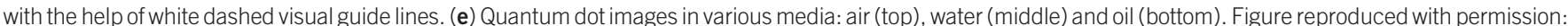
Ref. 8 ( 2013, National Academy of Sciences and Ref. 82 @ 2010, APS. SPP, surface plasmon polariton. 
a

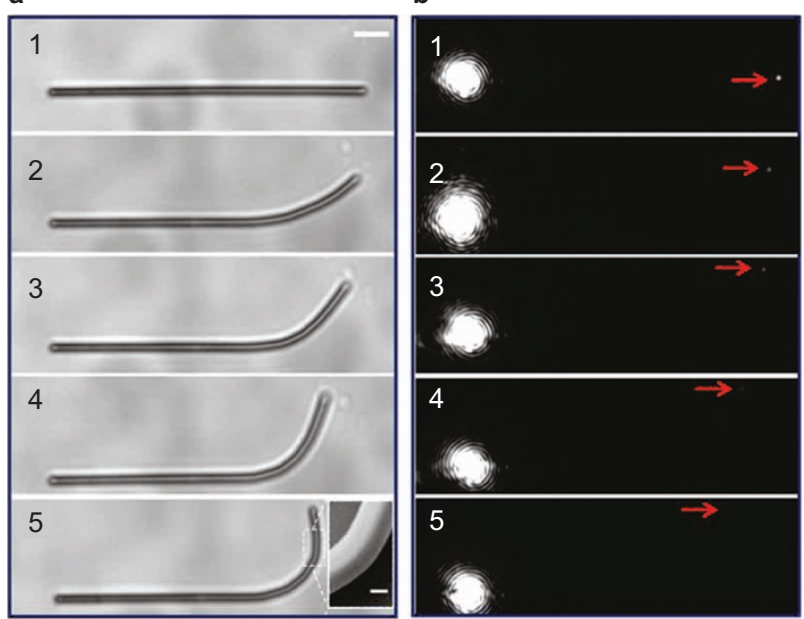

C

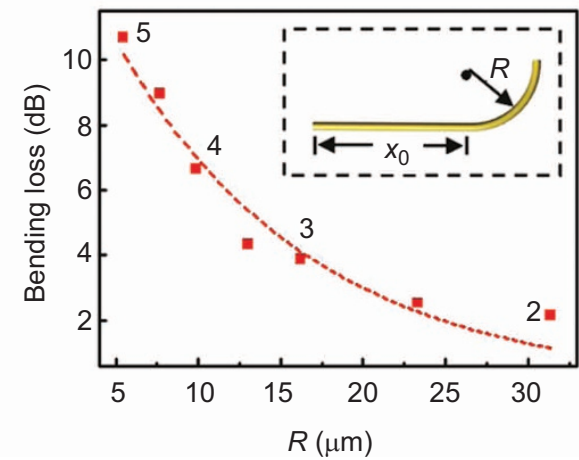

Figure 7 Plasmonic waveguide loss with various bending radii. (a) The bright field optical images of a 750-nm-diameter and 45- $\mu \mathrm{m}$-length Ag nanowire with different bending radii of $\infty, 32,16,9$ and $5 \mu \mathrm{m}$, respectively. The SEM image of the curved area is inset with a scale bar of $350 \mathrm{~nm}$. (b) The corresponding dark field images of the plasmonic waveguide. The emission at the distal bending terminal is illustrated by red arrows. (c) The measured values (red squares) and the exponential fitting (red line) of the pure bending loss as a function of bending radius. The inset is the geometry of the bending nanowire, where $R$ is the bending radius and $x_{0}$ is the straight length of the nanowire. Figure reproduced with permission: Ref. 84 (C) 2011, ACS. SEM, scanning electron microscopy.

served as a hotspot while being remotely excited by laser light with a wavelength of $632.8 \mathrm{~nm}$ (Figure 8).

A scanning electron microscopy (SEM) image of a nanoparticlenanowire system and a high magnification image of the Ag nanoparticle-nanowire junction are shown in Figure 8a. The corresponding optical bright field image, the optical image of the PSPPs, and the Raman image are shown in Figure $8 \mathrm{~b}, 8 \mathrm{c}, 8 \mathrm{~d}$ and $8 \mathrm{~g}$, where the laser was focused on one terminal of the nanowire, and the incident polarization is represented by the white arrow. The bright spot in Figure $8 \mathrm{c}$ shows the emitted photons from the PSPPs, whose propagation distance was approximately $6 \mu \mathrm{m}$. The image of the $436-\mathrm{cm}^{-1}$ Raman peak (Figure $8 \mathrm{~d}$ ) clearly shows that strong SERS signals were collected from the junction. Because of the single molecule level, the spectra in Figure 8e show the Raman signals obtained at the junction but not at the terminal of the nanowire. This could exclude the possibility that the Raman signal at the junction may come from the terminal via PSPPs. Eliminating the fluorescence background on the substrate in Figure 8f, the clear red spot in Figure $8 \mathrm{~g}$ shows the pure Raman signal of the MGITC at the single-molecule level with remote excitation (approximately $6 \mu \mathrm{m}$ away). The corresponding remote SERS spec- trum is presented in Figure 8h, which clearly shows the Raman features of malachite green isothiocyanate. If the focused laser spot deviated slightly from the terminal of the nanowire, but the other experimental conditions remained the same, there were no Raman signals from the junction. This fact could exclude the influence of the background scattering and confirm a real remote SERS. Similar experimental results in a very similar remote system were achieved by Hutchison and co-workers ${ }^{59}$ in the same year, where the Raman molecule was para-aminothiophenol.

\section{Single metal nanowire system}

Because of the simple structure, numerous studies on PSPPs in single noble nanowires have been reported since 2000 in an attempt to understand the corresponding physical mechanism. ${ }^{10,73-76}$ Remote SERS in a single metal nanowire system had also been investigated in 2011. We studied the remote SERS of MGITC and its polarization dependence on a single $\mathrm{Au}$ nanowire with a quasispherical terminal, which was excited by a laser with a wavelength of $632.8 \mathrm{~nm}$ (Figure 9). ${ }^{61}$ A sketch of this remote SERS experiment is illustrated in Figure 9a. The red, blue and orange arrows indicate the incident laser, PSPPs on the Au nanowire and the Raman scattering of MGITC on the terminal, respectively. The SEM image and the corresponding optical image in Figure $9 \mathrm{~b}$ and $9 \mathrm{c}$ indicate that a plasmonic waveguide was successfully achieved in an individual Au nanowire with a length of $6.5 \mu \mathrm{m}$ and a diameter of $210 \mathrm{~nm}$.

The remote SERS (red line) and local SERS (black line) spectra are shown in Figure 9d and demonstrate that the vibrational features of the malachite green isothiocyanate molecule were clearly depicted in both spectra, although the intensity of the remote SERS was approximately 150 times weaker than that of the local SERS. The remote SERS was also measured with different incident polarizations, and the maximal Raman signal was obtained, while the incident polarization was parallel to the nanowire. In such single nanowire remote systems, if the polarization of the target location needs to be set to a specific direction, it could be manipulated simply by changing the polarization of the incident light, but a measurement is needed in advance (Figure 10). ${ }^{76}$ Because of its anti-oxide property, a much longer working time under ambient environments could be achieved for Au plasmonic devices compared to Ag ones.

\section{Crossed metal nanowires system}

In the single nanowire system of remote SERS, the distance of the remote excitation is decided by the fixed length of the nanowire, and the Raman signal achieves a much lower level of enhancement. In nanoparticle-nanowire systems, although the hot spot at the nanogap formed by the nanoparticle adjacent to the nanowire was adjustable by varying the position of the nanoparticle along the nanowire and thus ensured the enhancement, the distance of the nanogap to the excitation end was not easily controlled experimentally. A crossed metal nanowire system formed by two nanowires at different directions may overcome the disadvantages mentioned above. The nanowire-nanowire junction of crossed nanowires as a hot spot could be readily positioned with a micromanipulator system, which could also be found easily with an optical microscope. Therefore, remote SERS in a crossed Ag nanowires system was investigated in our recent work. ${ }^{62}$

The spectra of remote SERS at a junction demonstrated that $p, p$ dimercaptoazobenzene (DMAB) could be generated from 4-nitrobenzenethiol (4NBT) induced by PSPPs, while an illumination laser with a wavelength of $632.8 \mathrm{~nm}$ was focused at the terminal of the nanowire (Figure 11). The SEM image and the corresponding optical image of 

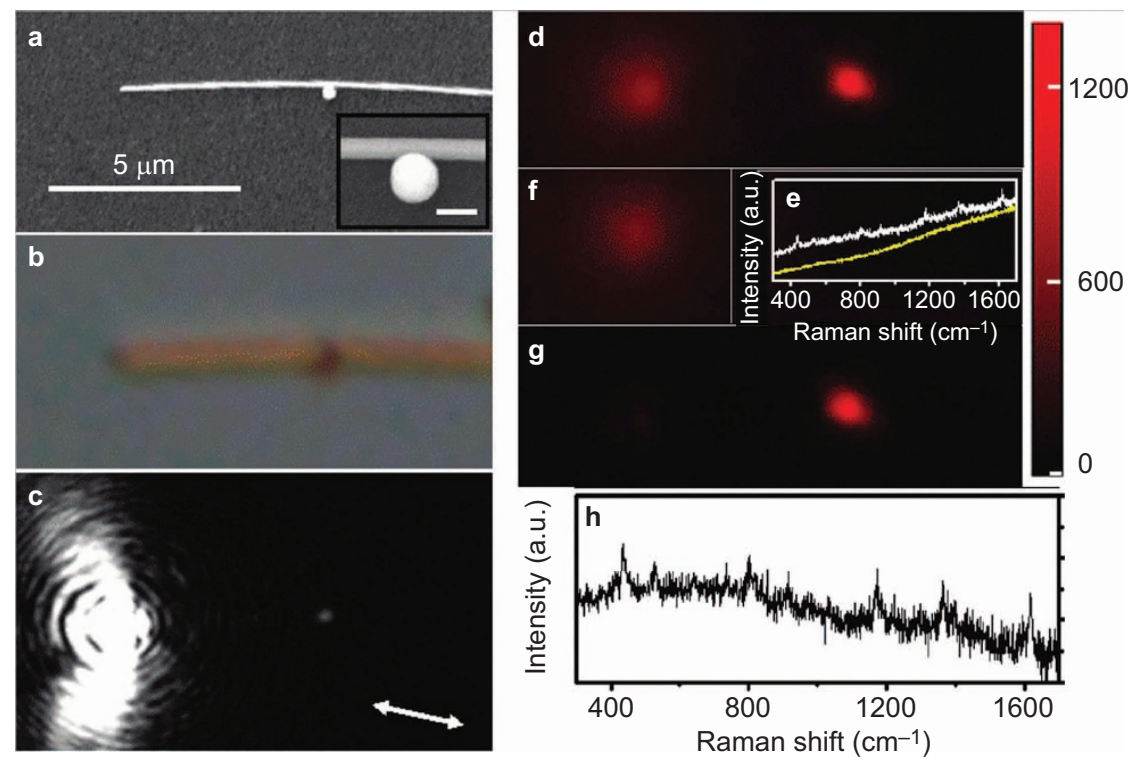

Figure 8 The SEM image (a) and the corresponding bright field image (b) of an Ag nanoparticle-nanowire system. (c) The dark field image of the plasmonic waveguide in this system. (d) The corresponding image of the $436 \mathrm{~cm}^{-1}$ Raman peak. (e) Local SERS (yellow line) and remote SERS (white line) spectra with fluorescence background. (f) The image of the fluorescence background at the laser spot. (g) The pure Raman image for the whole system. (h) The remote SERS spectrum without fluorescence background. Figure reproduced with permission: Ref. 60 ๑ 2009, ACS. SERS, surface-enhanced Raman scattering.

the PSPPs are shown in Figure 11a and 11b. The enlarged images of Figure 1la indicate that the diameters of the two crossed nanowires are 110 and $126 \mathrm{~nm}$, respectively. The tiny white spot at the junction indicates the coupling photons from the PSPPs while the laser was focused at the terminal of one nanowire approximately $3.6 \mu \mathrm{m}$ away. The obvious vibrational features of DMAB were represented by corresponding remote SERS spectra with different incident polarization in Figure 11d, which demonstrated that the surface catalysis reaction had been remotely induced by the PSPPs. The maximal intensity of the remote SERS was achieved while the incident polarization was parallel to the nanowire, which could be understood by the larger SPP propagating efficiency along the nanowire axis mentioned in the single Au nanowire system. To exclude the possibility that Raman signals obtained at the junction may have come from background scattering or the propagation of the SPPs at the illumination terminal, the corresponding Raman image at the peak of the $\mathrm{Ag}_{17}$ vibrational mode of the DMAB was measured, as shown in Figure 11c. The only color area, seen at the junction, meant that this remotely induced reaction only occurred in the hot spot because of the enhanced EM field. The time-dependent properties of this remote surface catalysis reaction in crossed Ag nanowires system was also confirmed in this work.

\section{Metal nanowire dimer system}

The nanowire dimer system includes end-to-end serially coupled nanowire pairs. The end-to-end junction formed by the terminals of different nanowires in this system could be the hot spot in the SERS measurement necessary to achieve a significantly enhanced Raman signal. Kumar's group has conducted interesting studies in local and remote SERS in an Ag nanowire dimer system in the last two years. ${ }^{63,87}$ In the previous reports on remote SERS, a single laser beam was focused through a microscope to excite PSPPs in remote systems, which could be called single-path excitation. In their reports on remote SERS in Ag nanowire dimer systems, two laser beams were simultaneously focused at the terminals of different nanowires, which was called dual-path excitation, to obtain the PSPPs that were eventually converted into radiating free photons at the end-to-end junction (Figure 12). The experimental and theoretical results indicated that stronger Raman signals could be obtained in dual-path excitation
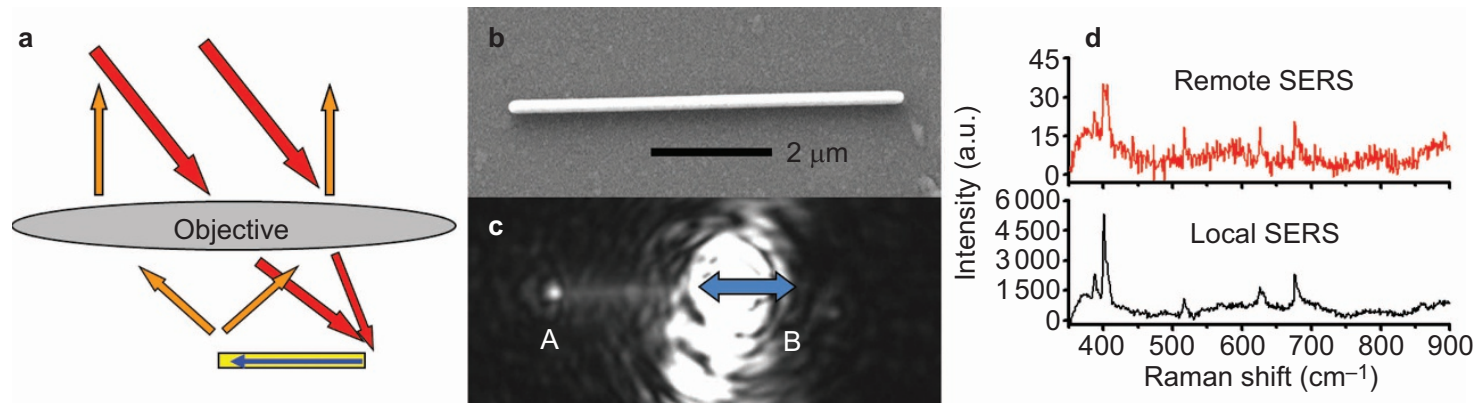

Figure 9 (a) The sketch of the remote SERS in a single Au nanowire. (b) The SEM image of a single Au nanowire. (c) The corresponding optical image of the plasmonic waveguide. (d) The top remote SERS spectrum (red line) obtained at terminus A and the bottom local SERS spectrum (black line) collected at terminus B. Figure reproduced with permission: Ref. 61 @ 2011, ACS. SEM, scanning electron microscopy; SERS, surface-enhanced Raman scattering. 
a
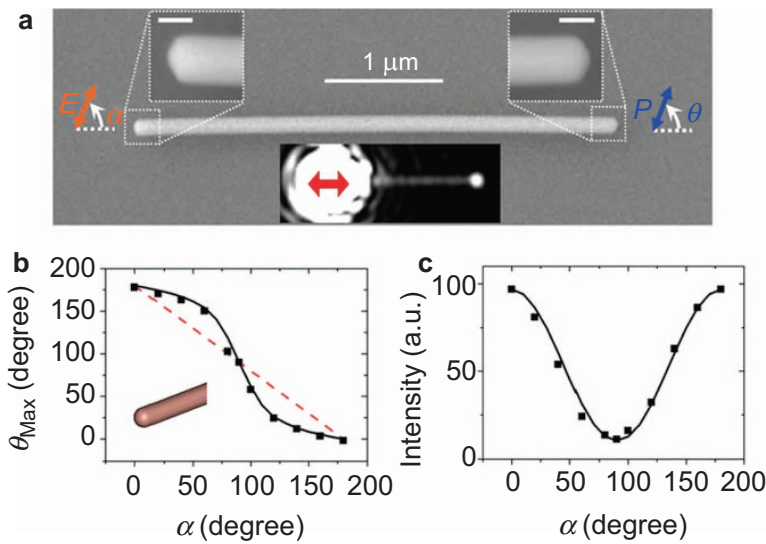

Figure 10 (a) The SEM image of a 148-nm-diameter and 4.54- $\mu$ m-length Ag nanowire and the corresponding dark field image of the plasmonic waveguide. The $\alpha$ (red arrow) and $\theta$ (blue arrow) indicate the polarization of the incident and emitted light, respectively. The polarization (b) and intensity (c) of the emitted light as a function of the incident polarization. Figure reproduced with permission: Ref. 76 @ 2013, ACS. SEM, scanning electron microscopy.
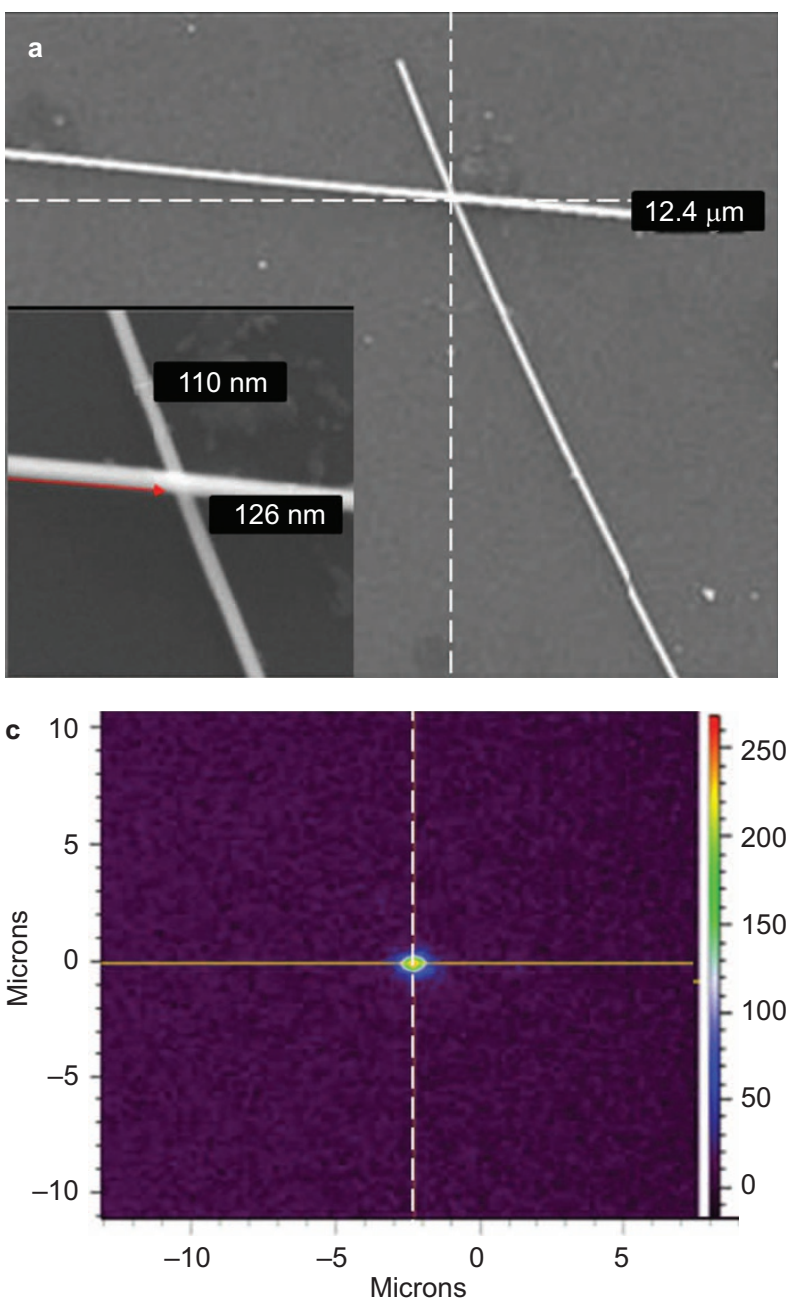

compared to that in single-path excitation at the same junction for equal values of excitation-laser powers.

Figure 12 shows the remote SERS spectra of Nile Blue-A at a junction with single- and dual-path excitation in the same Ag nanowire dimer system and the corresponding optical image of the PSPPs, in which the scattered light from the junction was visible as a bright red spot (indicated by a dotted circle). The two laser beams with wavelengths of $632.8 \mathrm{~nm}$, whose polarization are indicated as blue arrows in the images, were generated by a single laser beam with the help of a polarizing beam splitter. The two nanowires in this nanowire dimer system were 10- and $11-\mu \mathrm{m}$ long, having an obtuse angle of $150^{\circ}$ between them. Figure $12 \mathrm{a}$ and $12 \mathrm{~b}$ shows the experiment results with single-path remote excitation of end 1 or 2 , which were separately illuminated with a laser power of $8 \mathrm{~mW}$ with polarizations along the wires. In addition, Figure 12c illustrates the result with the dual-path remote excitation of both ends 1 and 2, which were simultaneously illuminated with a laser power of $4 \mathrm{~mW}$, each with the same polarizations. Interestingly, for the same excitation power ( $8 \mathrm{~mW}$ in total), the dual-path excitation (Figure 12c) resulted in stronger Raman signals compared to the single-path configurations (Figure 12a and 12b), which was approximately $70 \%$ larger according to the calculated integ-
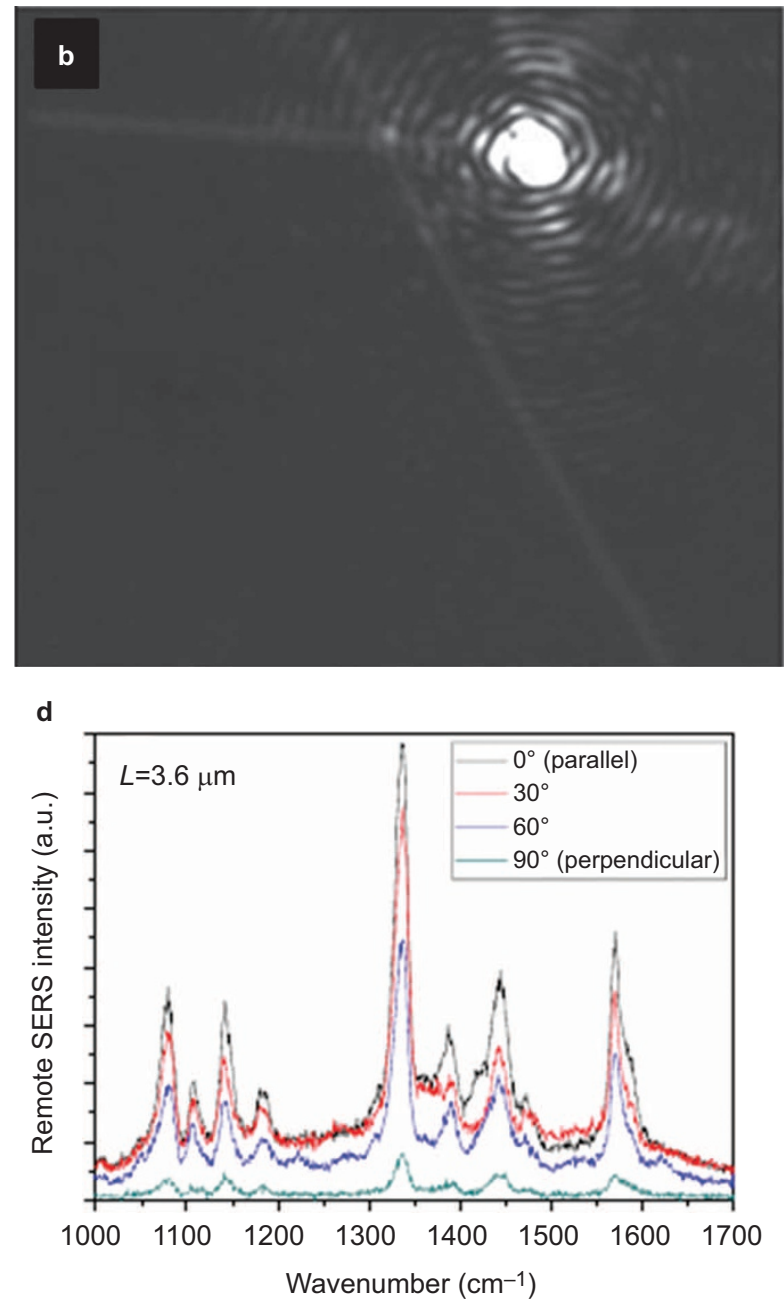

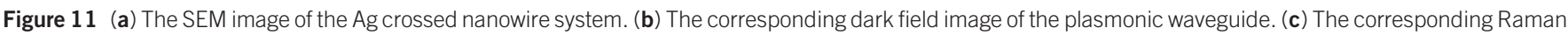

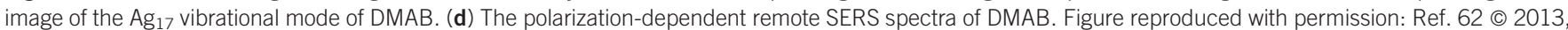
Springer. DMAB, $p, p$-dimercaptoazobenzene; SEM, scanning electron microscopy; SERS, surface-enhanced Raman scattering. 


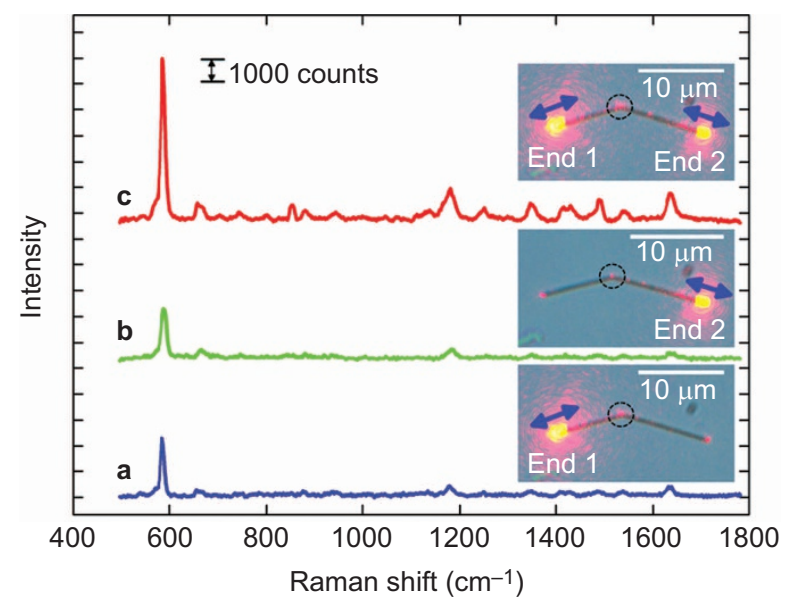

Figure 12 (a) Single-path remote SERS spectrum (blue line, excited at end 1). (b) Single-path remote SERS spectrum (green line, excited at end 2). (c) Dualpath remote SERS spectrum (red line, excited at both end 1 and end 2). The insets are the corresponding optical images of the plasmonic waveguide. Figure reproduced with permission: Ref. 63 (c) 2013, AIP. SERS, surface-enhanced Raman scattering.

ral intensity of the $590-\mathrm{cm}^{-1}$ peak in the spectra. The local SERS was also measured at the illuminating terminals, and the corresponding, albeit much fewer in numbers, detectable signals demonstrated that the collected remote SERS signals in the experiments were not Raman scattered light that had propagated from the illuminating terminals to the junction.

The polarization dependence of the remote SERS had also been investigated in the nanowire dimer system with dual-path excitation. The minimal Raman intensity was obtained, while both polarizations of the two illuminating laser beams were perpendicular to the nanowire individually. The maximal Raman intensity was achieved, while those were parallel to the nanowire individually. However, considering the interference of the two meeting PSPPs at the junction, the signal is not always the strongest when the system is excited by two beams from the same laser, but by controlling the phase difference of the two beams, strong or weak signals could be obtained up to the PSPPs from the two branches by coherent superposition or coherent cancellation. ${ }^{86} \mathrm{~A}$ similar study on dual-path excitation of remote SERS in an Ag nanoparticle-nanowire system has been reported by the same group. ${ }^{88}$ The enhanced Raman intensity from the dual-path excitation in these studies greatly improves the application of remote SERS.

\section{Metal nanowire bundles system}

The nanowire bundle system consists of a nanowire array produced by electrochemical deposition in a porous anodic alumina template. The large enhanced EM field in the tiny internanowire gaps of this system has numerous applications for plasmonics, such as plasmonicenhanced water splitting. ${ }^{39,40,89,90}$ Remote SERS in this system has also been recently report by Lee and Moskovits, ${ }^{64}$ in which the Ag nanowire bundles are treated as a probe. This probe could be attached to an optical fiber or a catheter to collect SERS signals in liquid or biotissue (Figure 13). The experiment setup and the structure of the probe are illustrated schematically at the top of Figure 13. The top of the anodic alumina film was slightly dissolved to ensure the attachment of the SERS molecule (4-aminobenzenethiol) to the Ag nanowire (Figure 13a). To reduce the reflection at the interface of the whole system and the glass substrate, an index matching adhesive was necessary for the SERS detection.
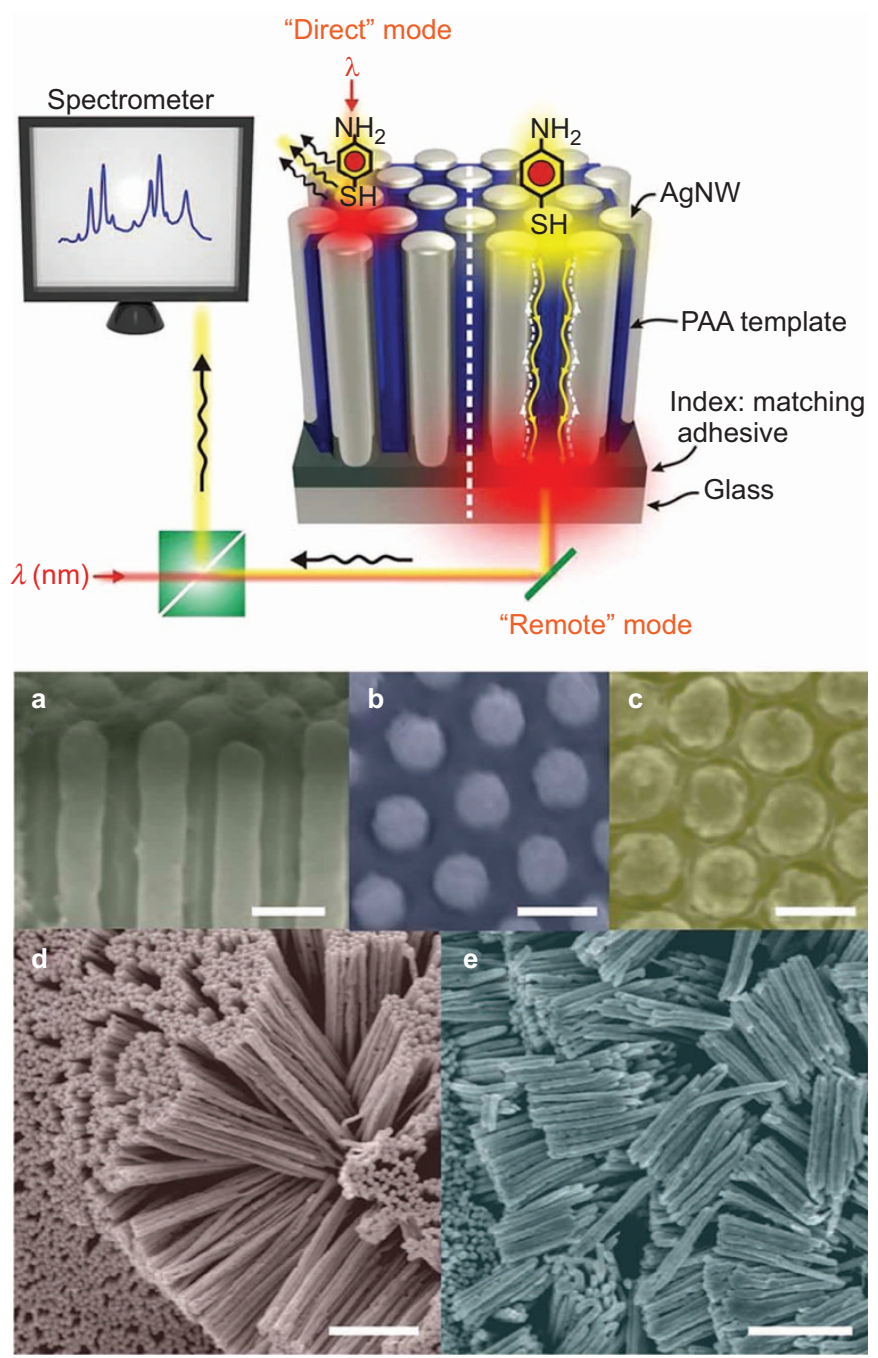

Figure 13 (Top) A sketch of the local and remote SERS in the Ag nanowire bundle system. (Bottom) The SEM images of the system comprising the sensor. (a) Lateral view of an Ag nanowire bundle system. (b) Top view of the system with 33-nm interwire gaps. (c) Top view of the system with 11-nm interwire gaps. (d, e) Systems with 2.2- $\mu$ m-length (33-nm gap) and 1- $\mu$ m-length (11-nm gap) after dissolving the porous alumina oxide template. Figure reproduced with permission: Ref. 64 () 2012, ACS. PAA, porous anodic alumina; SEM, scanning electron microscopy; SERS, surface-enhanced Raman scattering.

To study the diameter influence on the SERS signal, Ag bundles with 11- (Figure 13b) and 33-nm (Figure 13c) internanowire gap distances were prepared, while the center-to-center distance between the nanowires remained $100 \mathrm{~nm}$. The SEM images of Figure 13d and 13e indicated that the length of the Ag nanowire bundles was approximately $2.2 \mu \mathrm{m}$ (33-nm gap, 68-nm diameter) and approximately $1 \mu \mathrm{m}$ (11$\mathrm{nm}$ gap, $88-\mathrm{nm}$ diameter) individually. As shown in the sketch, the laser was directly illuminated on the top where the molecule was located for the local SERS (direct mode) and on the bottom (glass substrate) for the remote SERS (remote mode). Interestingly, in contrast to the previous remote SERS mentioned above, the PSPPs on the Ag nanowire were from the illuminating light as well as from the Raman scattering of molecules in the remote mode, which meant that the remote distance was two times the nanowire length. Largely, as a result of electrical resistance, the PSPPs along the Ag nanowire were attenuated exponentially as a function of the nanowire length. This 

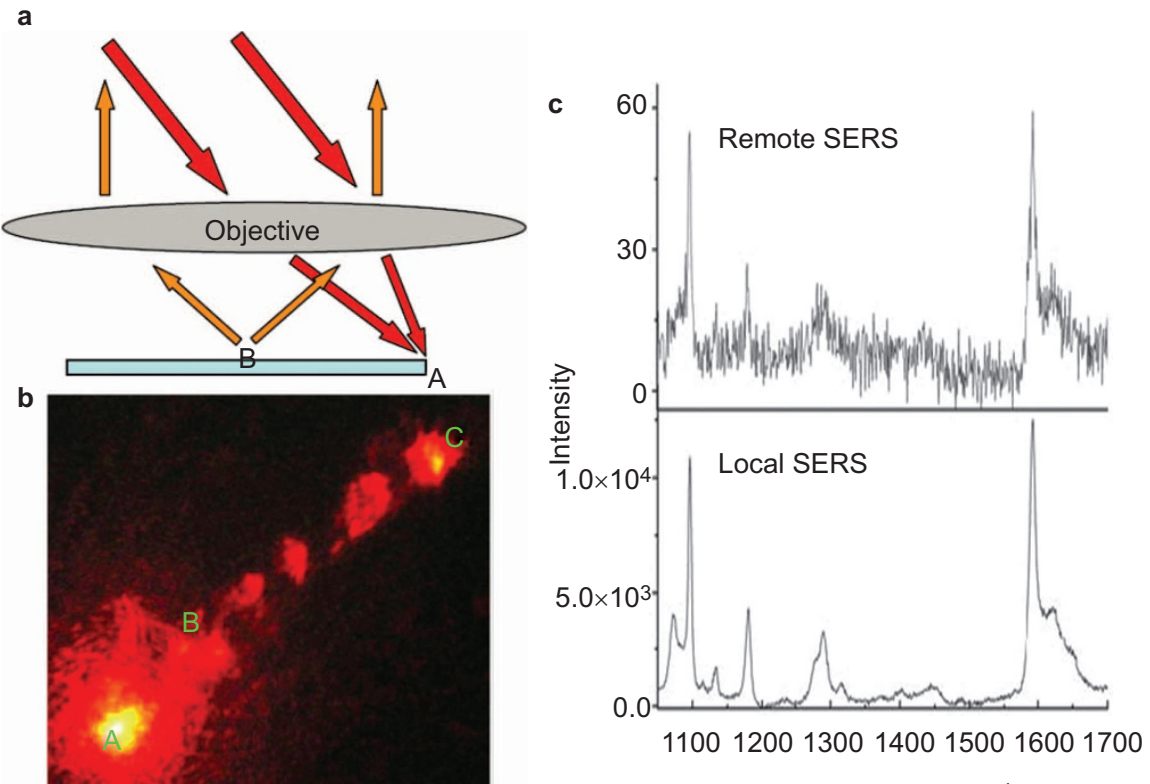

Raman shift $\left(\mathrm{cm}^{-1}\right)$

Figure 14 (a) A sketch of the remote SERS in a $\mathrm{MoO}_{3}$ belt. (b) The dark field image of the optical waveguide along the $\mathrm{MoO}_{3}$ belt. The whole length of this belt is approximately $52 \mathrm{~mm}$, while the distance from point A to point B is approximately $12.6 \mathrm{~mm}$. (c) The local SERS and remote SERS spectra of the 4-MBA molecule absorbed on the belt. Figure reproduced with permission: Ref. 49, ( 2010 Wiley-VCH. 4-MBA, 4-mercaptobenzoic acid; SERS, surface-enhanced Raman scattering.

fact was confirmed by the experimental results of the remote SERS in systems with nanowire lengths ranging from less than $1 \mu \mathrm{m}$ to over $3 \mu \mathrm{m}$. The results also indicated that a stronger Raman signal was obtained with a larger nanowire (88-nm diameter) compared to that with a smaller nanowire (68-nm diameter). This result was because the narrower internanowire gap functioned as better hot spots and because less attenuation was achieved by the larger nanowire.

\section{Semiconductor system}

Although the remote metal systems were dominant in studies of remote SERS, there were also several studies in semiconductor systems. We demonstrated that remote SERS was successfully performed using a dielectric waveguide in a quasi-one-dimensional (Q1D) $\mathrm{MoO}_{3}$ ribbon, not by PSPPs. ${ }^{49,65}$ Both remote SERS of 4 NBT in individual Q1D $\mathrm{MoO}_{3}$ ribbon systems (Figure 14$)^{49}$ and that in Ag nanoparticle-
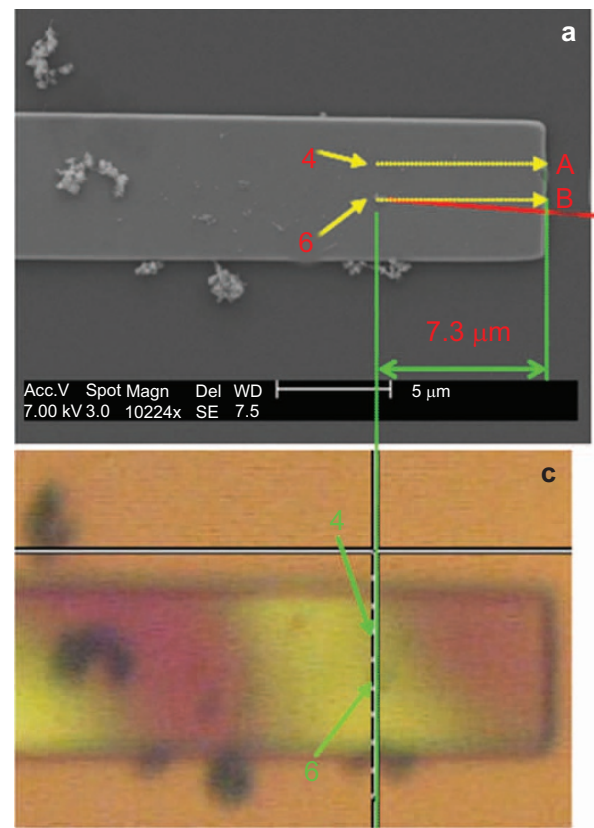
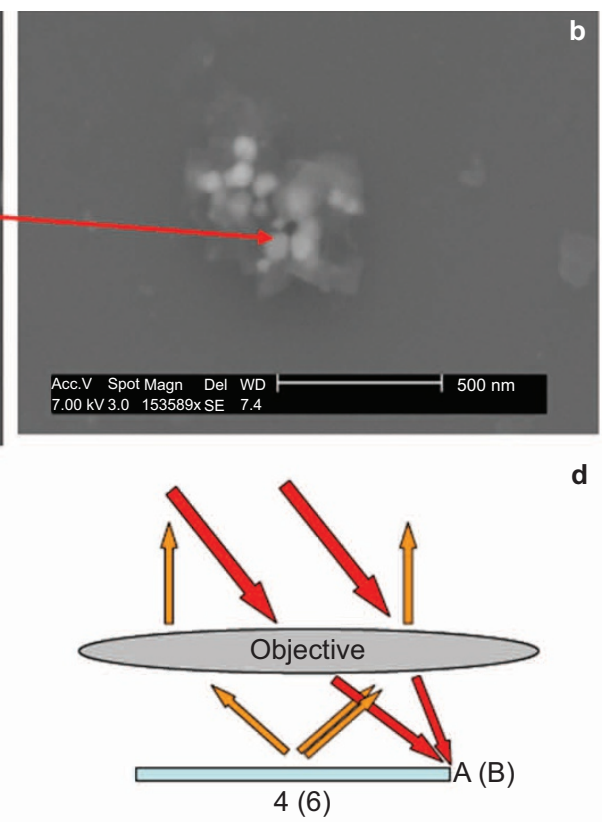
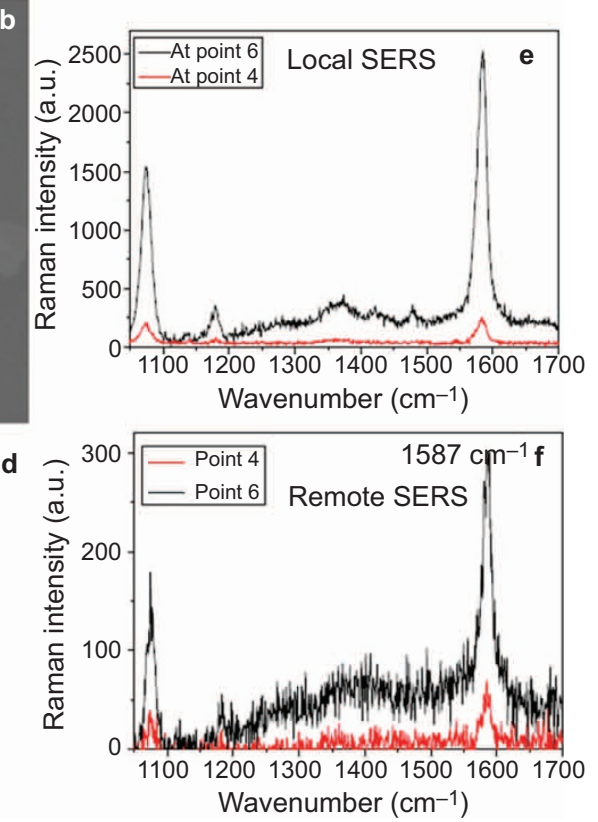

Figure 15 (a) The SEM image of the Ag nanoparticles and the $\mathrm{MoO}_{3}$ belt system. (b) The magnified SEM image of the Ag nanoparticles at point 6 in a. (c) The bright field optical image of the system. (d) The sketch of the remote SERS. (e) The local SERS spectra at points 4 and 6 in a. (f) The remote SERS spectra at points 4 and 6 in a. Figure reproduced with permission: Ref. 65 @ 2011, Springer. SEM, scanning electron microscopy; SERS, surface-enhanced Raman scattering. 

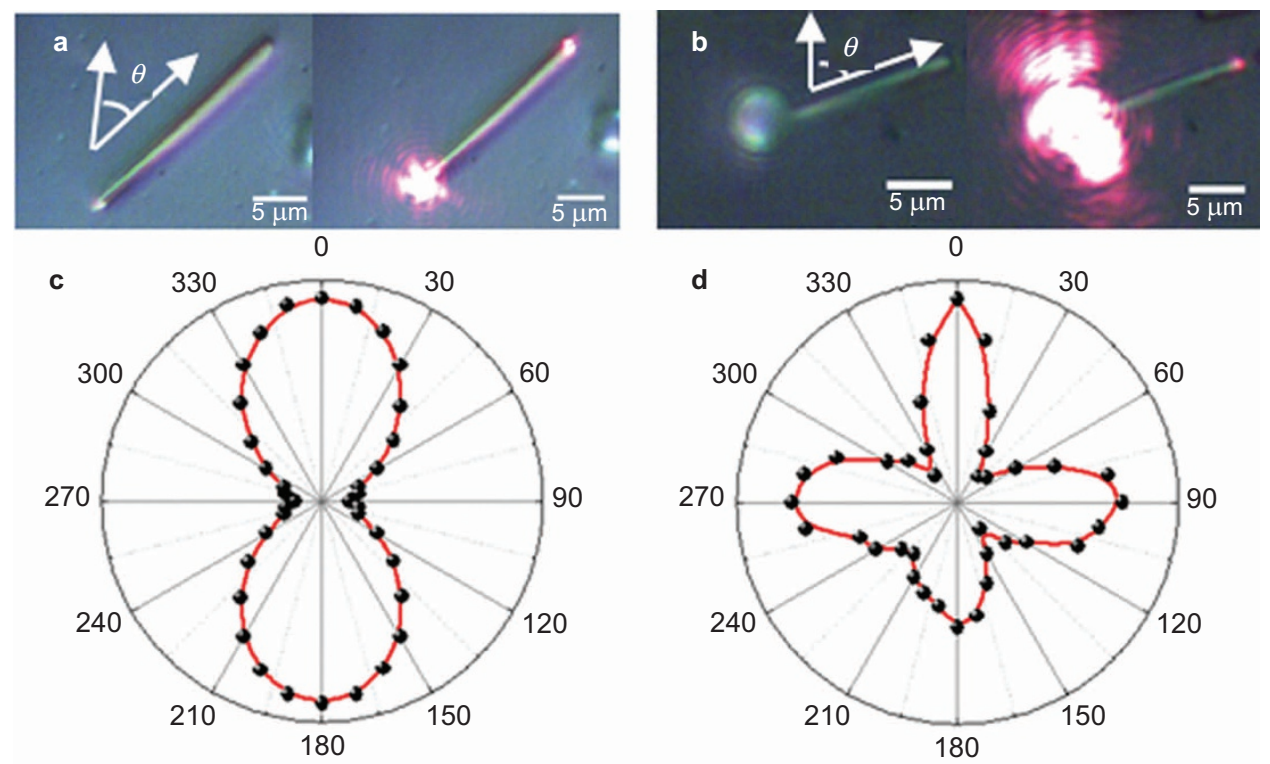

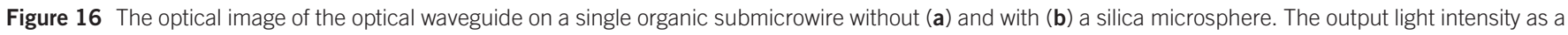
function of the input polarization without (c) and with (d) a silica microsphere. Figure reproduced with permission: Ref. 94 , (c) 2013 Wiley-VCH.

Q1D $\mathrm{MoO}_{3}$ ribbon system (Figure 15$)^{65}$ had been investigated, where the former exhibited chemical enhancement (charge transfer process) and the latter exhibited both chemical and physical (EM field) enhancement. A sketch of the remote SERS on an individual Q1D $\mathrm{MoO}_{3}$ ribbon is illustrated in Figure 14a. A laser with a wavelength of $632.8 \mathrm{~nm}$ was focused at one end of the Q1D $\mathrm{MoO}_{3}$ ribbon (A), and the SERS signal was collected on the other place $12.6 \mu \mathrm{m}$ away (B). The optical image in Figure 14b indicated this property of the dielectric waveguide on the Q1D $\mathrm{MoO}_{3}$ ribbon, whose length and width were $52 \mu \mathrm{m}$ and $3 \mu \mathrm{m}$. A similar feature of the corresponding Raman spectra in Figure $14 \mathrm{c}$ confirmed that the remote SERS was executed on the Q1D $\mathrm{MoO}_{3}$ ribbon, although its intensity was much weaker $(\sim 0.5 \%)$ compared to that of the local SERS.

The polarization dependence on the remote SERS of 4-mercaptobenzoic acid (4-MBA) was not observed in this experiment because the width of the ribbon was much larger than the wavelength of the laser. Similar results were achieved in the Ag nanoparticle-Q1D $\mathrm{MoO}_{3}$ ribbon as illustrated in Figure $15 .^{65}$ The SEM image and the corresponding optical image showed that the remote distance was $7.3 \mu \mathrm{m}$. The laser was focused at the terminal of the Q1D $\mathrm{MoO}_{3}$ ribbon (A and B), while the Raman signals were collected at points 4 and 6, where the Ag nanoparticles was located as shown in the sketch in Figure 15d. The spectra of the local and remote SERS are presented in Figure 15e and 15f. The stronger Raman intensity at point 6 was a result of the hot spots formed by the aggregation of Ag nanoparticles, which is shown in Figure 15b. Both the chemical and EM field mechanism contributed to the remote SERS in this study. The former came from the charge transfer between the $4 \mathrm{NBT}$ and the $\mathrm{MoO}_{3}$ ribbon, and the latter arose from the SPPs of the Ag nanoparticles whose energy was transferred by the propagating dielectric waveguide on the Q1D $\mathrm{MoO}_{3}$ ribbon.

\section{Organic system}

The optical waveguide could be realized in metal and semiconductor systems as well as in an organic system. The studies of optical waveguides on one-dimensional organic submicron-structures has been an interesting topic in recent years for their potential future applications in the areas of nano-/microscale organic photonic devices, sensors, light-concentrating devices, waveguides, etc. ${ }^{91-93}$ A typical optical waveguide in an organic system is illustrated in Figure 16, which is performed on a submicrowire assembled by a 1,5-diaminoanthraquinone molecule. ${ }^{94}$ The substantial influence on the output light intensity due to the existence of silica microspheres indicates that the structure also plays a key role in this waveguide. Recently, remote SERS has also been achieved on self-assembled organic submicrotubes with various geometries (linear, C-bent, X-ben and tip-to-tip) (Figure 17). ${ }^{66}$ The diameter and length of the tubes in this study were $0.8-1.5 \mu \mathrm{m}$ and 7-400 $\mu \mathrm{m}$, respectively. The low Rayleigh and scattered Raman light had been collected at one terminal of the organic tube while a laser with a different wavelength had been focused at the distal terminal. Unlike in the remote metal system, the remote Raman signal appeared not only at the terminal but also at the other position along the organic tubes. This result was obtained because the momentum mismatch mentioned in the plasmonic waveguide is not needed in both organic and dielectric systems based on traditional optical waveguides.

Figure 17 illustrates the remote SERS in three types of organic tubes (I represents the linear tube, II the bent one and III the crossed tubes, as shown in the bright field images in Figure 17a). The wavelength of the excited laser was $532 \mathrm{~nm}$ and had a spot size of approximately $5 \mu \mathrm{m}$. The red and blue circles represent the input and output light areas. It was very clear that the optical waveguide had been realized in all types of organic system in Figure 17b (bright field image) and 17c (dark field image). Equipped with a 570-nm long-pass edge filter, the distributions of low Rayleigh and Raman photons were 3D mapped, as shown in Figure $17 \mathrm{~d}$ and $17 \mathrm{e}$. The image of a strong $1609-\mathrm{cm}^{-1}(\mathrm{C}=\mathrm{C}$ stretch) Raman peak indicated the optical waveguide path along the organic tubes. Dividing by the input intensity, the output intensity of light was estimated as a function of time. A preferential interaction between input photons and molecules, generating more Raman signals, was revealed by the experimental result and indicated that the Raman intensity was much stronger than the low Rayleigh intensity. The difference in the Raman intensity between the linear and bent 

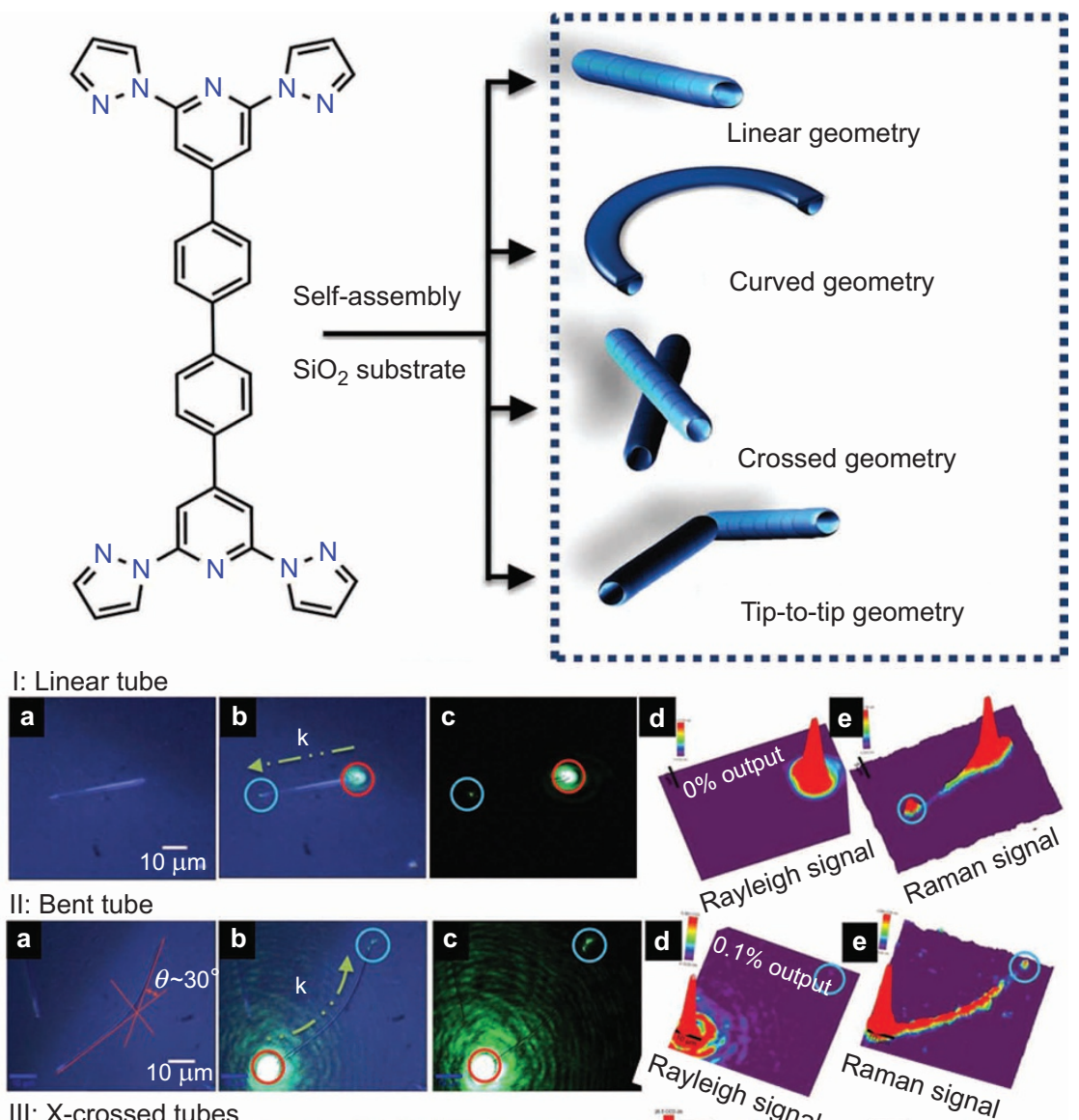

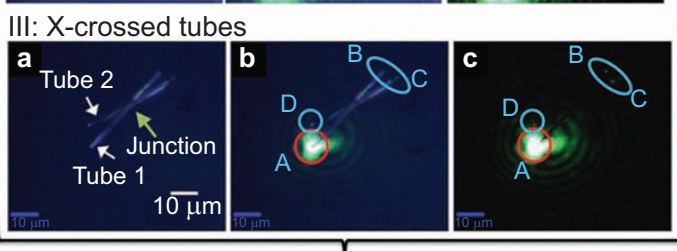

Without $570 \mathrm{~nm}$ LPEF

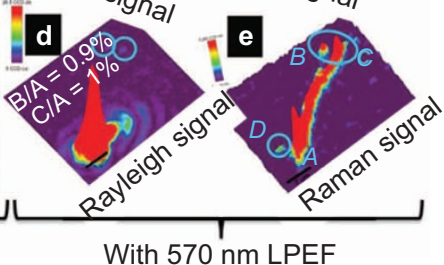

Figure 17 (Top) The structure of the organic molecule and the corresponding self-assembled organic submicrotubes with different geometries. (Bottom) The remote SERS in organic tubes. (a) The bright field images of organic tubes. (b, c) The bright field and dark field images of the optical waveguide in tubes. (d, e) The image of low Rayleigh and Raman scattering. The input and scattering light are indicated by blue and red circles. Figure reproduced with permission: Ref. 66, (0) 2013 Wiley-VCH. LPEF, long-pass edge filter; SERS, surface-enhanced Raman scattering.

tubes explains the variation of the molecular environment on tubes. The strong Raman signal exhibited for the bent tubes was a result of the favorable molecular orientation and defect sites caused by bending.

\section{APPLICATIONS}

As a novel technology invented in only 5 years, fascinating properties of remote SERS have already been applied in several fields, such as sensitive sensors, plasmon-driven surface-catalyzed reactions and Raman optical activity.

\section{Sensor for in vivo cells}

Because of the sensitivity at the single molecule level and the resolution at the nanoscale, sensitive sensors are the most frequently mentioned application of remote SERS in previous reports. ${ }^{59-61,63,64}$ Specifically, it has been treated as a novel, powerful sensing technology to monitor bioreactions in vivo in cells. ${ }^{59,60}$ Although this interesting design has not been realized yet, we introduce some exploratory work here to provide a method of improvement. In 2012, Yang's group ${ }^{67}$ reported nanowire based endoscopy in single in vivo cells (Figure 18). As shown in Figure 18a, this endoscopy consists of $\mathrm{anO}_{2}$ nanowire attached to the tapered tip of an optical fiber. With the help of a three-axis micromanipulating system, the inserted nanowire could be the optical waveguide supporter at designated positions in a single in vivo cell. The optical coupling through a nanowire could be treated as either a local light source for a subcellular image or as a spectrometer for local optical signal collection. Using the nanowire endoscope, the schematics of the subcellular image and the local fluorescence of quantum dots (emission, $655 \mathrm{~nm}$ ) in an in vivo cell are presented in Figure $18 \mathrm{~b}$ and $18 \mathrm{c}$. In the remote detection of the subcellular spectrum, the nanowire was located near the preloaded quantum dot, which was excited by a blue light $(442 \mathrm{~nm})$. The fluorescence of the quantum dot was then collected through the nanowire coupling to a spectrometer. The dark field image in Figure $18 \mathrm{~d}$ and the remote collected fluorescence spectrum with a single peak at $655 \mathrm{~nm}$ in Figure $18 \mathrm{e}$ confirmed the realization of remote spectrum detection in vivo in cells. 

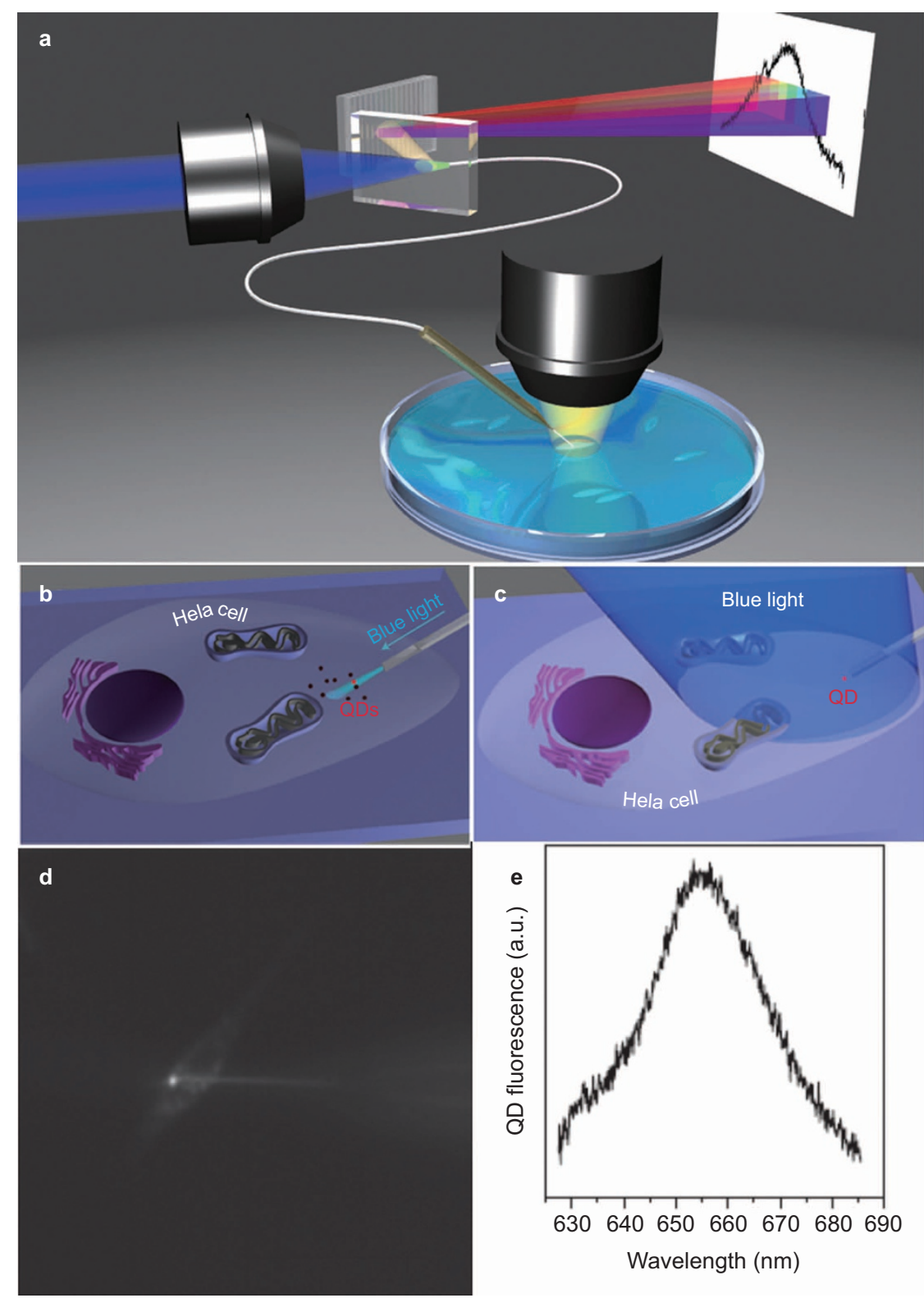

Figure 18 (a) A sketch of the nanowire endoscope system for single in vivo cells. (b, c) A sketch of subcellular imaging and local spectrum remotely collected in an in vivo cell through the system. (d) The dark field image of the remote detection of quantum dot fluorescence in an in vivo cell. (e) The corresponding spectrum remotely collected through the nanowire end scope system. Figure reproduced with permission: Ref. 67 @ 2013, NPG. QD, quantum dot.

This result has great significance to the application of remote SERS for in vivo cells such that we can adopt similar equipment with some improvement so that the excitation and detection could occur simultaneously. Light emission at the terminal of a nanowire based on a waveguide could be treated as the excitation source, and the Raman spectra could also be collected by the spectrometer through the same nanowire based on a waveguide. To achieve a greater intensity of the spectra, the semiconductor nanowire could be replaced by the noble metal nanowire for the electromagnetic enhancement of SPPs. We are sure that this remote SERS device has broad application prospects for monitoring bioreactions in vivo in cells.

\section{Plasmon-driven surface-catalyzed reactions}

Surface catalysis reactions of 4NBT or para-aminothiophenol converting to DMAB could be induced by SPPs, which are also called plasmon-driven surface-catalyzed reactions. This reaction was first reported theoretically in 2010 and subsequently demonstrated in a series of studies by local SERS. ${ }^{95-98}$ Furthermore, remote SERS was introduced into this surface catalysis reaction in our previous reports, where the PSPPs were carriers for both catalysis and sensing. ${ }^{62,68,69} \mathrm{We}$ demonstrated for the first time in 2011 that DMAB could be produced by PATA at Ag nanoparticle-nanowire junctions through remote SERS technology in the nanoparticle-nanowire system (Figure 19). ${ }^{69}$ The SEM image and optical image of the PSPPs present in Figure 19a and 19b indicated that the remote distance was $3.4 \mu \mathrm{m}$ and the diameter of the nanowire that the SPPs propagated on was $130 \mathrm{~nm}$. The polarization of the $632.8 \mathrm{~nm}$ incident laser was parallel to the nanowire to obtain a maximal signal. The tiny white spot at the junction indicates light radiation converted by PSPPs from the distal terminal of the nanowire illuminated by the laser. 

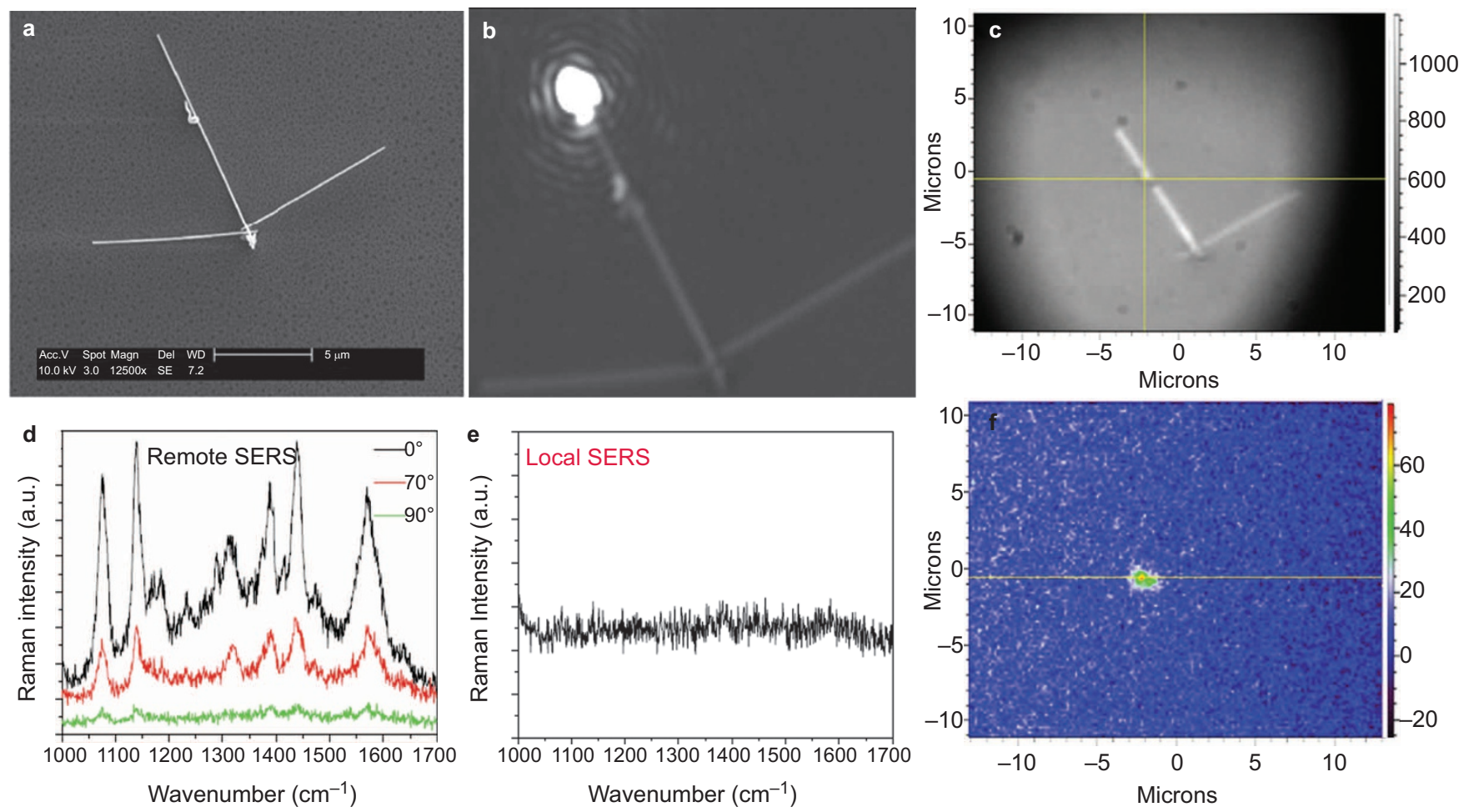

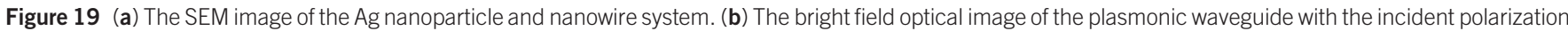
along the nanowire to obtain the maximal signal. (d) The incident polarization dependence of the remote SERS spectra in this system. (e) The local SERS spectrum

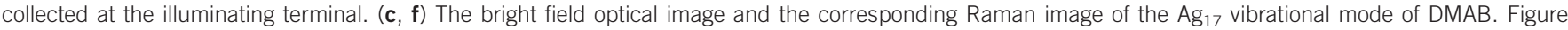

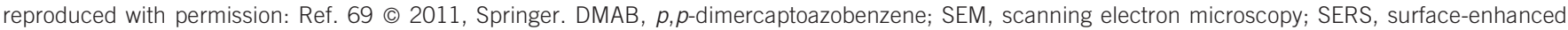
Raman scattering.

The measured remote SERS spectra at the junction in Figure 19d exhibited the Raman feature of DMAB and demonstrated that the plasmon-driven surface-catalyzed reaction did occur. The intensity fluctuation of different incident polarizations in the spectra could be understood by the polarization dependence of the PSPPs mentioned above. The local SERS spectrum in Figure 19e shows that the plasmondriven surface-catalyzed reaction did not take place at the illuminating terminal of the nanowire. This was because of the much weaker enhanced EM field at the illuminating terminal compared to that at the junction, which was essential in the plasmon-driven surface-catalyzed reactions. The Raman image of the $\mathrm{Ag}_{17}$ vibrational mode of the DMAB in Figure 19f and the corresponding optical image in Figure 19c confirmed that this surface catalysis occurred only at the junction through remote SERS.

\section{Raman optical activity}

The Raman optical activity (ROA) resulted in differences in the Raman spectra excited by right- and left-circularly polarized light, which is ultrasensitive to the chirality of the molecule structure and therefore indicates that this activity could reveal the absolute molecular configuration and conformation. ${ }^{99,100}$ Although this amazing phenomenon is widely used in biological technology, such as chiral-sensitive vibrational spectroscopy, the weak ROA intensity becomes the bottleneck of applications, which is almost $10^{-3}-10^{-5}$ times the intensities of the parent Raman scattering. ${ }^{101}$ SERS was reported by several groups to enhance ROA for the extremely enhanced EM field. ${ }^{102-108}$ We have demonstrated that this enhancement could also be realized through remote SERS of fmoc-glycyl-glycine-OH in an $\mathrm{Ag}$ nanowire system in rapid communications (Figure 20). ${ }^{70}$ The SEM image and the optical image of the PSPPs present in Figure 20a and 20b indicated that the crossing angle between the nanowires was determined to be approximately $20^{\circ}$ and released light at the terminals of the nanowire. The local and remote Raman images of fmoc-glycyl-glycine-OH at $1593 \mathrm{~cm}^{-1}$, excited by left- and right-circularly polarized light, are shown in Figure 20c and 20d, respectively. This vibrational mode exhibited large Raman and ROA intensities without the influence of chemical enhancement. Due to the chiral properties of the nanowire structure and the PSPPs, they exhibited different local and remote intensities of the Raman imaging at $1593 \mathrm{~cm}^{-1}$. It was obvious that the Raman intensities at four terminals were much stronger while being excited by the left-circular polarization.

To quantify the ROA, the dimensionless circular intensity differences (CIDs) at different terminals, which was defined as the result of the difference between the Raman scattering intensities of right- and left-circularly polarized light divided by their sum, are presented in Figure 20e. The CIDs were significantly enhanced compared to the intrinsic molecular powder, which was approximately $0.57 \%$. Furthermore, different CIDs were obtained at three remote terminals (b, $c, d)$. The small difference between the CID at the illuminating terminal a and that at terminal $b$ (the distal terminal of the same Ag nanowire) indicated that the chirality of both the PSPPs and the molecule did not change in an individual nanowire. However, the larger difference between CIDs at terminals of another nanowire (c or d) and the illuminating terminal showed that the CID could be manipulated by the chirality of a nanostructure, such as through the coupling angle between two Ag nanowires, the morphology of the Ag terminals, and the distance of the coupling. ${ }^{6-9}$ In this system, the CID increased while the crossing angle was less than $90^{\circ}$ (terminal c) and 

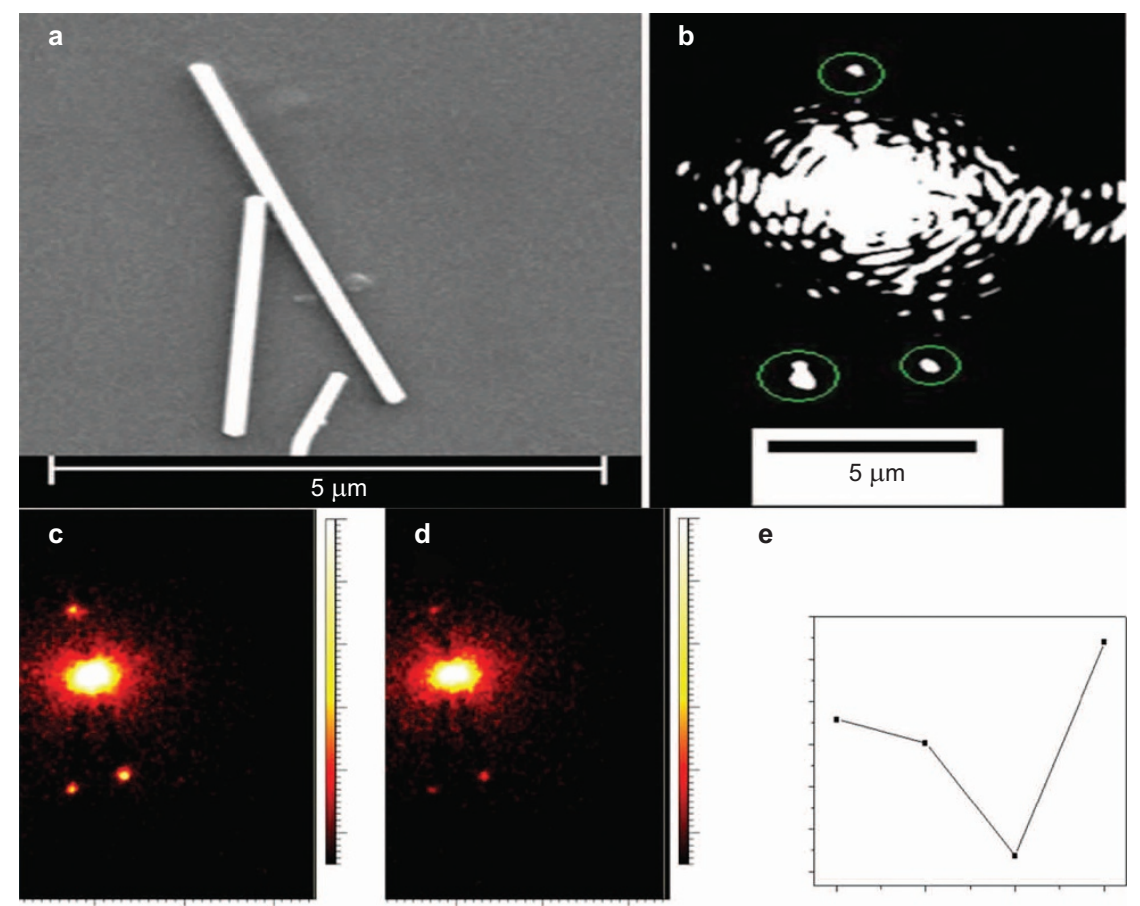

Figure 20 (a) The SEM image of the Ag nanowires. (b) The corresponding dark field image of the plasmonic waveguide. (c, d) The corresponding Raman images excited by left- or right-circularly polarization light. (e) The fitted CIDs at four points in the Ag nanowires in a. Figure reproduced with permission: Ref. 70 ( 2013 , NPG. CID, circular intensity difference; SEM, scanning electron microscopy.

decreased, while the crossing angle was larger than $90^{\circ}$. These experimental results demonstrated that the ROA could be remotely excited and enhanced by chiral PSPPs, which had the potential to remotely determine the molecular chirality or the absolute configuration or conformation of a chiral living cell.

\section{CONCLUSION AND OUTLOOK}

Combining propagating and localized SPPs, remote SERS was first realized experimentally in 2009. Significant progress has been made to further support and develops this novel technology. Various remote systems have been investigated to enlarge its application field, including metal systems based on plasmonic waveguides, semiconductors and organic systems based on dielectric waveguides. In remote metal systems, such as the Ag nanoparticle-nanowire system, the large EM field at the hot spot that is coupled to the PSPPs could effectively enhance the intensity of remote SERS to achieve single molecule levels. This useful property of remote SERS presents a new approach to sensing surface-enhanced optical signals in special environments where common optical sensing technology is unsuitable, such as Raman detection in vivo in cells.

In the Ag nanowire system, the chirality of the PSPPs on the Ag nanowire in remote SERS, which could be affected by the coupling angle between two Ag nanowires, the morphology of the Ag terminals, the distance of the coupling, etc., makes the modulation of enhanced ROA of chiral molecules a reality. In an Ag crossed nanowire system, the enhanced EM field at a hot spot in the remote SERS could improve the surface catalysis reaction of converting 4NBT or para-aminothiophenol to DMAB, which introduces a new way to synthesis molecules at the nanoscale. Using an optical waveguide in a $\mathrm{Q} 1 \mathrm{D} \mathrm{MoO}_{3}$ ribbon dielectric and in organic submicrotubes, remote SERS has been achieved in semiconductor and organic systems, which demonstrates another method of realizing remote SERS without plasmonic waveguides. Therefore, it is expected that further studies on remote SERS could be performed in various remote systems that consist of the materials mentioned above as well as other suitable ones, such as graphene, $\mathrm{ZnO}$ and $\mathrm{Si}$.

\section{ACKNOWLEDGEMENTS}

This study was financially supported by the National Natural Science Foundation of China (Grants 11374353, 11204390 and 11274149), the Fundamental Research Funds for the Central Universities (CQDXWL-2013009), the Program of Shenyang Key Laboratory of Optoelectronic Materials and Technology (F12-254-1-00) and Special Fund for Agro-scientific Research in the Public Interest (NO. 201303045).

1 Halas NJ, Lal S, Chang WS, Link S, Nordlander P. Plasmons in strongly coupled metallic nanostructures. Chem Rev 2011; 111: 3913-3961.

2 Mayer KM, Hafner JH. Localized surface plasmon resonance sensors. Chem Rev 2011; 111: 3828-3857.

3 Ozbay E. Plasmonics: merging photonics and electronics at nanoscale dimensions. Science 2006; 311: 189-193.

4 Giannini V, Fernández-Domínguez Al, Heck SC, Maier SA. Plasmonic nanoantennas: fundamentals and their use in controlling the radiative properties of nanoemitters. Chem Rev 2011; 111: 3888-3912.

5 Lal S, Hafner JH, Halas NJ, Link S, Nordlander P. Noble metal nanowires: from plasmon waveguides to passive and active devices. Accounts Chem Res 2012; 45: 1887-1895.

6 Wei H, Li ZP, Tian XR, Wang ZX, Cong FZ et al. Quantum dot-based local field imaging reveals plasmon-based interferometric logic in silver nanowire networks. Nano Lett 2011; 11: 471-475.

7 Wei H, Wang ZX, Tian XR, Käll M, Xu HX. Cascaded logic gates in nanophotonic plasmon networks. Nat Commun 2011; 2: 387.

8 Wei H, Zhang SR, Tian XR, Xu HX. Highly tunable propagating surface plasmons on supported silver nanowires. Proc Natl Acad Sci USA 2013; 110: 4494-4499.

9 Zhang SP, Wei H, Bao K, Hakånson U, Halas NJ et al. Chiral surface plasmon polaritons on metallic nanowires. Phys Rev Lett 2011; 107: 096801.

10 Fang YR, Li ZP, Huang YZ, Zhang SP, Nordlander P et al. Branched silver nanowires as controllable plasmon routers. Nano Lett 2010; 10: 1950-1954. 
11 Huang LL, Chen XZ, Bai BF, Tan QF, Jin GF et al. Helicity dependent directional surface plasmon polariton excitation using a metasurface with interfacial phase discontinuity. Light Sci App/2013; 2: e70; doi:10.1038/lsa.2013.26.

12 Wei H, Xu HX. Hot spots in different metal nanostructures for plasmon-enhanced Raman spectroscopy. Nanoscale 2013; 5: 10794-10805.

13 Kneipp K, Wang Y, Kneipp H, Perelman LT, Itzkan I et al. Single molecule detection using surface-enhanced Raman scattering (SERS). Phys Rev Lett 1997; 78: 16671670.

14 Xu HX, Bjerneld EJ, Käll M, Börjesson L. Spectroscopy of single hemoglobin molecules by surface enhanced Raman scattering. Phys Rev Lett 1999; 83: 4357-4360.

15 Lozano G, Louwers DJ, Rodríguez SR, Murai S, Jansen OT et al. Plasmonics for solidstate lighting: enhanced excitation and directional emission of highly efficient light sources. Light Sci App/ 2013; 2: e66; doi:10.1038/lsa.2013.22.

16 Zhang SP, Bao K, Halas NJ, Xu HX, Nordlander P. Substrate-induced Fano resonances of a plasmonic nanocube: a route to increased-sensitivity localized surface plasmon resonance sensors revealed. Nano Lett 2011; 11: 1657-1663.

17 Käll M. Plasmonic nanosensors: inverse sensitivity. Nat Mater 2012; 11: 570-571.

18 Liu N, Hentschel M, Weiss T, Alivisatos AP, Giessen H. Three-dimensional plasmon rulers. Science 2011; 332: 1407-1410.

19 Sun Q, Ueno K, Yu H, Kubo A, Matsuo Y et al. Direct imaging of the near field and dynamics of surface plasmon resonance on gold nanostructures using photoemission electron microscopy. Light Sci App/ 2013; 2: e118; doi:10.1038/Isa.2013.74.

20 Lezec HJ, Degiron A, Devaux E, Linke RA, Martin-Moreno L et al. Beaming light from a subwavelength aperture. Science 2002; 297: 820-822.

21 Sun S, Yang KY, Wang CM, Juan TK, Chen WT et al. High-efficiency broadband anomalous reflection by gradient meta-surfaces. Nano Lett 2012; 12: 6223-6229.

22 Juan ML, Righini M, Quidant R. Plasmon nano-optical tweezers. Nat Photonics 2011; 5: 349-356.

23 Roxworthy BJ, Ko KD, Kumar A, Fung KH, Chow EK et al. Application of plasmonic bowtie nanoantenna arrays for optical trapping, stacking, and sorting. Nano Lett 2012; 12: 796-801.

24 Yoo J, Ma X, Tang W, Yi GC. Metal-lined semiconductor nanotubes for surface plasmon-mediated luminescence enhancement. Nano Lett 2013; 13: 2134-2140.

25 Zhang W, Ding F, Chou SY. Large enhancement of upconversion luminescence of $\mathrm{NaYF}_{4}: \mathrm{Yb}^{3+} / \mathrm{Er}^{3+}$ nanocrystal by 3D plasmonic nano-antennas. Adv Mater 2012; 24: OP236-OP241.

26 Marinica DC, Kazansky AK, Nordlander P, Aizpurua J, Borisov AG. Quantum plasmonics: nonlinear effects in the field enhancement of a plasmonic nanoparticle dimer. Nano Lett 2012; 12: 1333-1339.

27 Akimov AV, Mukherjee A, Yu CL, Chang DE, Zibrov AS et al. Generation of single optical plasmons in metallic nanowires coupled to quantum dots. Nature 2007; 450: 402-406.

28 Fang N, Lee H, Sun C, Zhang X. Sub-diffraction-limited optical imaging with a silver superlens. Science 2005; 308: 534-537.

29 Ergin T, Stenger N, Brenner P, Pendry JB, Wegener M. Three-dimensional invisibility cloak at optical wavelengths. Science 2010; 328: 337-339.

30 Hirsch LR, Stafford RJ, Bankson JA, Sershen SR, Rivera B et al. Nanoshell-mediated near-infrared thermal therapy of tumors under magnetic resonance guidance. Proc Natl Acad Sci USA 2003; 100: 13549-13554.

31 Loo C, Lowery A, Halas N, West J, Drezek R. Immunotargeted nanoshells for integrated cancer imaging and therapy. Nano Lett 2005; 5: 709-711.

32 Neumann O, Urban AS, Day J, Lal S, Nordlander P et al. Solar vapor generation enabled by nanoparticles. ACS Nano 2013; 7: 42-49.

33 Fang ZY, Zhen YR, Neumann O, Polman A, García de Abajo FJ et al. Evolution of lightinduced vapor generation at a liquid-immersed metallic nanoparticle. Nano Lett 2013; 13: 1736-1742.

34 Ozaki M, Kato J, Kawata S. Surface-plasmon holography with white-light illumination. Science 2011; 332: 218-220.

35 Atwater HA, Polman A. Plasmonics for improved photovoltaic devices. Nat Mater 2010; 9: 205-213.

36 Polman A, Atwater HA. Photonic design principles for ultrahigh-efficiency photovoltaics. Nat Mater 2012; 11: 174-177.

37 Su YH, Ke YF, Cai SL, Yao QY. Surface plasmon resonance of layer-by-layer gold nanoparticles induced photoelectric current in environmentally-friendly plasmonsensitized solar cell. Light Sci App/ 2012; 1: e14; doi:10.1038/lsa.2012.14.

38 Chalabi H, Brongersma ML. Plasmonics: harvest season for hot electrons. Nat Nanotechnol 2013; 8: 229-230.

39 Moskovits M. Hot electrons cross boundaries. Science 2011; 332: 676-677.

40 Mubeen S, Lee J, Singh N, Krämer S, Stucky GD et al. An autonomous photosynthetic device in which all charge carriers derive from surface plasmons. Nat Nanotechnol 2013; 8: 247-251.

41 Fleischmann M, Hendra PJ, Mcquillan AJ. Raman-spectra of pyridine adsorbed at a silver electrode. Chem Phys Lett 1974; 26: 163-166.

42 Jeanmaire DL, Vanduyne RP. Surface Raman spectroelectrochemistry. 1. Heterocyclic, aromatic, and aliphatic-amines adsorbed on anodized silver electrode. J Electroanal Chem 1977; 84: 1-20.

43 Metiu H, Das P. The electromagnetic theory of surface enhanced spectroscopy. Annu Rev Phys Chem 1984; 35: 507-536.

44 Moskovits M. Surface-enhanced spectroscopy. Rev Mod Phys 1985; 57: 783-826.

45 Lombardi JR, Birke RL. A unified view of surface-enhanced Raman scattering. Accounts Chem Res 2009; 40: 734-742.

46 Zhao L, Jensen L, Schatz GC. Pyridine-Ag20 cluster: a model system for studying surface-enhanced Raman scattering. J Am Chem Soc 2006; 128: 2911-2919.
47 Otto A. The 'chemical' (electronic) contribution to surface-enhanced Raman scattering. J Raman Spectrosc 2005; 36: 497-509.

48 Xia LX, Chen MD, Zhao XM, Zhang ZL, Xia JR et al. Visualized method of chemical enhancement mechanism on SERS and TERS. J Raman Spectrosc 2014; 45: 533540 .

49 Dong B, Huang YZ, Yu NS, Fang YR, Cao BS et al. Local and remote charge-transferenhanced Raman scattering on one-dimensional transition-metal oxides. Chem Asian J 2010; 5: 1824-1829.

50 Huang YZ, Fang YR, Yang ZL, Sun MT. Can $p, p^{\prime}$-dimercaptoazobisbenzene be produced from $p$-aminothiophenol by surface photochemistry reaction in the junctions of a Ag nanoparticle-molecule-Ag (or Au) film? J Phys Chem C 2010; 114: 18263-18269.

51 Sun MT, Huang YZ, Xia LX, Chen XW, Xu HX. The $\mathrm{pH}$-controlled plasmon-assisted surface photocatalysis reaction of 4-aminothiophenol to $p, p^{\prime}$-dimercaptoazobenzene on Au, Ag, and Cu colloids. J Phys Chem C 2011; 115: 9629-9636.

52 Huang YZ, Dong B. pH dependent plasmon-driven surface-catalysis reactions of $p, p^{\prime}$ dimercaptoazobenzene produced from para-aminothiophenol and 4-nitrobenzenethiol. Sci China Chem 2012; 55: 2567-2572.

53 Fang YR, Huang YZ. Electromagnetic field redistribution in hybridized plasmonic particle-film system. App/ Phys Lett 2013; 102: 153108.

54 Dong B, Liu LW, Xu HX, Sun MT. Experimental and theoretical evidence for the chemical mechanism in SERRS of rhodamine $6 \mathrm{G}$ adsorbed on colloidal silver excited at $1064 \mathrm{~nm}$. J Raman Spectrosc 2010; 41: 719-720.

55 Sun MT, Fang YR, Zhang Z, Xu HX. Activated vibrational modes and Fermi resonance in tip-enhanced Raman spectroscopy. Phys Rev E 2013; 87: 020401(R).

56 Li JF, Huang YF, Ding Y, Yang ZL, Li SB et al. Shell-isolated nanoparticle-enhanced Raman spectroscopy. Nature 2010; 464: 392-395.

57 Gómez-Graña S, Pérez-Juste J, Alvarez-Puebla RA, Guerrero-Martínez A, Liz-Marzán LM. Self-assembly of Au@Ag nanorods mediated by gemini surfactants for highly efficient SERS-active supercrystals. Adv Opt Mater 2013; 1: 477-481.

58 Wei H, Hao F, Huang YZ, Wang WZ, Nordlander P et al. Polarization dependence of surface-enhanced Raman scattering in gold nanoparticle-nanowire systems. Nano Lett 2008; 8: 2497-2502.

59 Hutchison JA, Centeno SP, Odaka H, Fukumura H, Hofkens J et al. Subdiffraction limited, remote excitation of surface enhanced Raman scattering. Nano Lett 2009; 9 995-1001.

60 Fang YR, Wei H, Hao F, Nordlander P, Xu HX. Remote-excitation surface-enhanced Raman scattering using propagating Ag nanowire plasmons. Nano Lett 2009; 9: 2049-2053.

61 Huang YZ, Fang YR, Sun MT. Remote excitation of surface-enhanced Raman scattering on single Au nanowire with quasi-spherical termini. J Phys Chem C $2011 ; 115$ : 3558-3561.

62 Song P, Li YZ, Li YQ, Zhao MY, Liu LW et al. Remote-excitation time-dependent surface catalysis reaction using plasmonic waveguide on sites of single-crystalline crossed nanowires. Plasmonics 2013; 8: 249-254.

63 Dasgupta A, Singh D, Kumar GV. Dual-path remote-excitation surface enhanced Raman microscopy with plasmonic nanowire dimer. Appl Phys Lett 2013; 103 : 151114.

64 Lee SJ, Moskovits M. Remote sensing by plasmonic transport. J Am Chem Soc 2012; 134: 11384-11387.

65 Dong B, Zhang W, Li ZP, Sun MT. Remote excitation surface plasmon and consequent enhancement of surface-enhanced Raman scattering using evanescent wave propagating in quasi-one-dimensional $\mathrm{MoO}_{3}$ ribbon dielectric waveguide. Plasmonics 2011; 6: 189-193.

66 Chandrasekhar N, Mohiddon MA, Chandrasekar R. Organic submicro tubular optical waveguides: self-assembly, diverse geometries, efficiency, and remote sensing properties. Adv Opt Mater 2013; 1: 305-311.

67 Yan RX, Park JH, Choi Y, Heo CJ, Yang SM et al. Nanowire-based single-cell endoscopy. Nat Nanotechnol 2012; 7: 191-196.

68 Sun MT, Hou YX, Xu HX. Can information of chemical reaction propagate with plasmonic waveguide and be detected at remote terminal of nanowire? Nanoscale 2011; 3: 4114-4116.

69 Sun MT, Hou YX, Li ZP, Liu LW, Xu HX. Remote excitation polarization-dependent surface photochemical reaction by plasmonic waveguide. Plasmonics 2011; 6: 681687.

70 Sun MT, Zhang ZL, Wang PJ, Li Q, Ma FC et al. Remotely excited Raman optical activity using chiral plasmon propagation in Ag nanowires. Light Sci App/ 2013; 2: e112; doi:10.1038/lsa.2013.68.

71 Bozhevolnyi SI, Volkov VS, Devaux E, Laluet JY, Ebbesen TW. Channel plasmon subwavelength waveguide components including interferometers and ring resonators. Nature 2006; 440: 508-511.

72 Apuzzo A, Février M, Salas-Montiel R, Bruyant A, Chelnokov A et al. Observation of near-field dipolar interactions involved in a metal nanoparticle chain waveguide. Nano Lett 2013; 13: 1000-1006.

73 Dickson RM, Lyon LA. Unidirectional plasmon propagation in metallic nanowires. J Phys Chem B 2000; 104: 6095-6098.

74 Ditlbacher H, Hohenau A, Wagner D, Kreibig U, Rogers $M$ et al. Silver nanowires as surface plasmon resonators. Phys Rev Lett 2005; 95: 257403.

75 Li ZP, Hao F, Huang YZ, Fang YR, Nordlander P et al. Directional light emission from propagating surface plasmons of silver nanowires. Nano Lett 2009; 9: 4383-4386.

76 Li ZP, Bao K, Fang YR, Huang YZ, Nordlander P et al. Correlation between incident and emission polarization in nanowire surface plasmon waveguides. Nano Lett 2010; 10: $1831-1835$ 
77 Shegai T, Huang YZ, Xu HX, Käll M. Coloring fluorescence emission with silver nanowires. Appl Phys Lett 2010; 96: 103114.

78 Zhang SP, Chen L, Huang YZ, Xu HX. Reduced linewidth multipolar plasmon resonances in metal nanorods and related applications. Nanoscale 2013; 5: 6985-6991.

79 Guo X, Ma YG, Wang YP, Tong LM. Nanowire plasmonic waveguides, circuits and devices. Laser Photonics Rev 2013; 7: 855-881.

80 Xia Y, Xiong Y, Lim B, Skrabalak SE. Shape-controlled synthesis of metal nanocrystals: simple chemistry meets complex physics? Angew Chem 2009; 48: 60-103.

81 Kusar P, Gruber C, Hohenau A, Krenn JR. Measurement and reduction of damping in plasmonic nanowires. Nano Lett 2012; 12: 661-665.

82 Li ZP, Bao K, Fang YR, Guan ZQ, Halas NJ et al. Effect of a proximal substrate on plasmon propagation in silver nanowires. Phys Rev B 2010; 82: 241402(R).

83 Chang DE, Sørensen AS, Hemmer PR, Lukin MD. Strong coupling of single emitters to surface plasmons. Phys Rev B 2007; 76: 035420.

84 Wang WH, Yang Q, Fan FR, Xu HX, Wang ZL. Light propagation in curved silver nanowire plasmonic waveguides. Nano Lett 2011; 11: 1603-1608.

85 Knight MW, Grady NK, Bardhan R, Hao F, Nordlander P et al. Nanoparticle-mediated coupling of light into a nanowire. Nano Lett 2007; 7: 2346-2350.

86 Li ZP, Zhang SP, Halas NJ, Nordlander P, Xu HX. Coherent modulation of propagating plasmons in silver-nanowire-based structures. Small 2011; 7: 593-596.

87 Chikkaraddy R, Singh D, Kumar GV. Plasmon assisted light propagation and Raman scattering hot-spot in end-to-end coupled silver nanowire pairs. Appl Phys Lett 2012 100: 043108

88 Dasgupta A, Singh D, Tandon S, Tripathi RP, Kumar GV. Remote-excitation surfaceenhanced Raman scattering with counter-propagating plasmons: silver nanowirenanoparticle system. J Nanophotonics 2013; 8: 083899.

89 Lee J, Mubeen S, Ji X, Stucky GD, Moskovits M. Plasmonic photoanodes for solar water splitting with visible light. Nano Lett 2012; 12: 5014-5019.

90 Mubeen S, Hernandez-Sosa G, Moses D, Lee J, Moskovits M. Plasmonic photosensitization of a wide band gap semiconductor: converting plasmons to charge carriers. Nano Lett 2011; 11: 5548-5552.

91 Wang P, Wang YP, Tong LM. Functionalized polymer nanofibers: a versatile platform for manipulating light at the nanoscale. Light Sci App/2013; 2: e102; doi:10.1038/ Isa.2013.58.

92 Zhao YS, Fu H, Peng A, Ma Y, Liao Q et al. Construction and optoelectronic properties of organic one-dimensional nanostructures. Acc Chem Res 2010; 43: 409-418.

93 Chandrasekhar N, Chandrasekar R. Reversibly shape-shifting organic optica waveguides: formation of organic nanorings, nanotubes, and nanosheets. Angew Chem 2012; 51: 3556-3561.

94 Chikkaraddy R, Dasgupta A, Gupta SD, Kumar GV. Microsphere-coupled organic waveguides: preparation, remote excitation of whispering gallery modes and waveguiding property. Appl Phys Lett 2013; 103: 031112.
95 Sun MT, Xu HX. A novel application of plasmonics: plasmon-driven surface-catalyzed reactions. Small 2012; 8: 2777-2786.

96 Fang YR, Li YZ, Xu HX, Sun MT. Ascertaining p, p'-dimercaptoazobenzene produced from $p$-aminothiophenol by selective catalytic coupling reaction on silver nanoparticles. Langmuir 2010; 26: 7737-7746.

97 Dong B, Fang YR, Chen XW, Xu HX, Sun MT. Substrate-, wavelength-, and timedependent plasmon-assisted surface catalysis reaction of 4-nitrobenzenethiol dimerizing to $p, p^{\prime}$-dimercaptoazobenzene on Au, Ag, and Cu films. Langmuir 2011; 27: 10677-10682.

98 Sun MT, Zhang ZL, Zheng HR, Xu HX. In-situ plasmon-driven chemical reactions revealed by high vacuum tip-enhanced Raman spectroscopy. Sci Rep 2012; 2: 647.

99 Barron LD, Buckingham AD. Rayleigh and Raman scattering from optically active molecules. Mol Phys 1971; 20: 1111-1119.

100 Barron LD, Bogaard MP, Buckingham AD. Raman-scattering of circularly polarizedlight by optically-active molecules. J Am Chem Soc 1973; 95: 603-605.

101 Hug W, Kint S, Bailey GF, Scherer JR. Raman circular intensity differential spectroscopy. Spectra of (-)-.alpha.-pinene and (+)-.alpha.-phenylethylamine. J Am Chem Soc 1975; 97: 5589-5590.

102 Efrima S. The effect of large electric field gradients on the Raman optical activity of molecules adsorbed on metal surfaces. Chem Phys Lett 1983; 102: 79-82.

103 Efrima S. Raman optical activity of molecules adsorbed on metal surfaces: theory. J Chem Phys 1985; 83: 1356-1362.

104 Etchegoin PG, Galloway C, Le Ru EC. Polarization-dependent effects in surfaceenhanced Raman scattering (SERS). Phys Chem Chem Phys 2006; 8: 2624-2628.

105 Janesko BG, Scuseria GE. Surface enhanced Raman optical activity of molecules on orientationally averaged substrates: theory of electromagnetic effects. J Chem Phys 2006; 125: 124704

106 Kneipp H, Kneipp J, Kneipp K. Surface-enhanced Raman optical activity on adenine in silver colloidal solution. Anal Chem 2006; 78: 1363-1366.

107 Abdali S, Blanch EW. Surface enhanced Raman optical activity (SEROA). Chem Soc Rev 2008; 37: 980-992.

108 Novák V, Šebestík J, Bouř P. Theoretical modeling of the surface-enhanced Raman optical activity. J Chem Theory Comput 2012; 8: 1714-1720.

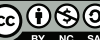

This work is licensed under a Creative Commons Attribution-

NonCommercial-ShareAlike 3.0 Unported License. The images or other third party material in this article are included in the article's Creative Commons license, unless indicated otherwise in the credit line; if the material is not included under the Creative Commons license, users will need to obtain permission from the license holder to reproduce the material. To view a copy of this license, visit http://creativecommons.org/licenses/by-nc-sa/3.0/ 